Article

\title{
A meaning for the second power of the number Pi. Determining the surface of a regular conoid by virtue of Ramanujan's approximation
}

\author{
Jose M. Cabeza- Lainez ${ }^{1 *}$ \\ 1 Higher Technical School of Architecture, University of Seville, 41012, Seville, Spain; crowley@us.es. \\ Member of the Japan Society for the Promotion of Science in Spain. \\ * Correspondence: crowley@us.es; Tel.: +34-696 749344
}

\begin{abstract}
: unlike the volume, the expression for the lateral area of a regular conoid has not yet been obtained by means of direct integration or a differential geometry procedure. As this form is relatively used in engineering, the inability to determine its surface, represents a serious hindrance for several problems which arise in radiative transfer, lighting and construction, to cite just a few. Since this particular shape can be conceived as a set of linearly dwindling ellipses which remain parallel to a circular directrix, a typical problem appears when looking for the length of such ellipses. We conceived a new procedure which, in principle, consists in dividing the surface into infinitesimal elliptic strips to which we have subsequently applied Ramanujan's second approximation. In this fashion, we can obtain the perimeter of any ellipse pertaining to the said form as a function of the radius of the directrix and the position of the ellipse's center on the X-axis. Integrating the so-found perimeters of the differential strips for the whole span of the conoid, an unexpected solution emerges through the newly found number psi $(\psi)$. As the strips are slanted in the symmetry axis, their width is not uniform and we need to perform some adjustments in order to complete the problem with sufficient precision. Relevant implications for technology, building science, radiation and structure are derived in the ensuing discussion.
\end{abstract}

Keywords: Conoid; Ellipse; Ramanujan; Calculus of surface areas; number Psi; number Pi; 3Dconstruction of complex Geometries; Engineering Design Objects. Architectural Forms.

\section{Introduction}

\subsection{Outline of the problem}

Since antiquity the meaning of the number Pi has been associated to the length of a circumference, that is, such length, if the diameter of the said circumference is the unit, equates $\mathrm{Pi}$, and correspondingly for different measures of the diameter. Even in the Bible, when injunctions are transferred to build an offering's laver (the sea of bronze) of circular device in Solomon's Temple, it is mentioned that the perimeter ought to be three times the diameter [1] a revealing estimate.

This said, should not we speculate, from a scientific point of view, about the meaning of different powers of $\mathrm{Pi}$, like for example, $\mathrm{Pi}^{\mathrm{N}}$ ? 
The answer is Yes, and in this article we would discuss the particular case of the second power of $\pi$, that is, Pi squared or $\mathrm{Pi}^{2}$. Such situation arises when we try to calculate the surface area of a Conoid, a ruled surface generated by parallel straight lines which project from a circumference directrix onto a linear edge (Fig.1).

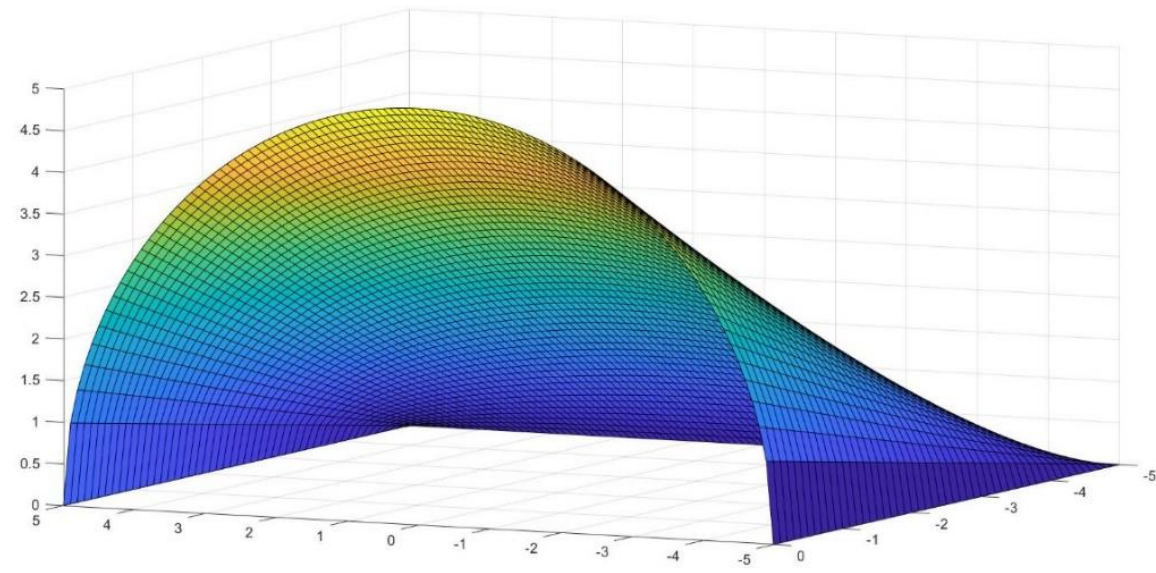

Figure 1. A typical straight conoid with circular directrix, where $R=L=5$.

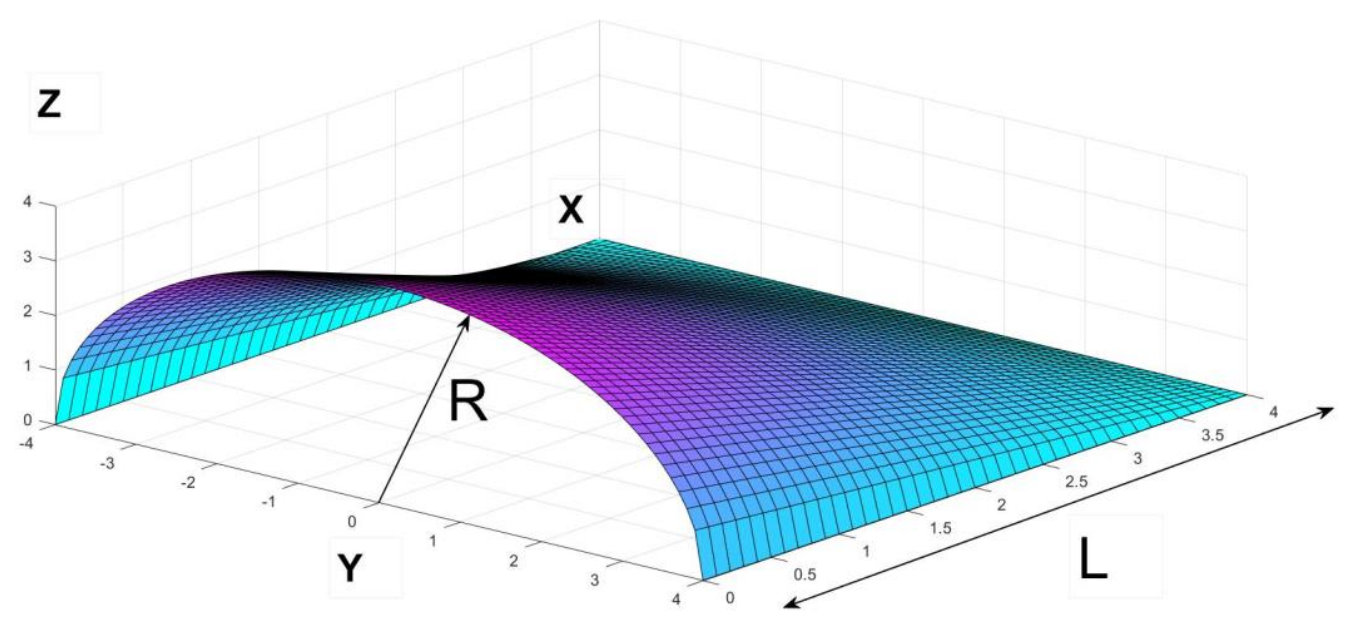

Figure 2. Explanation of the parts of a straight conoid with circular directrix, in this case $R=L=4$.

The equation that regulates such warped figure for $\mathrm{x}$ and $\mathrm{y}$ positive is:

$$
\frac{L^{2} z^{2}}{(L-x)^{2}}+y^{2}=R^{2}
$$

Where $R$ is the radius of the directrix in the case of a circumference and $L$ is the length in the $X$ direction as shown in figure 2. If we make $L=R$, it turns out that, 


$$
\frac{R^{2} z^{2}}{(R-x)^{2}}+y^{2}=R^{2}
$$

To solve the problem of the lateral area of such surface, after not reaching a conclusion with other methods, we would employ Ramanujan's second approximation for ellipses. [2]

\section{Methods and materials}

\subsection{Resolution of the proposed approximation}

In his well-known proposal to calculate the perimeter of an ellipse, Ramanujan stated that the perimeter $(\mathrm{P})$ of the curve equates:

$$
\mathrm{P}=\pi(3(a+b)-\sqrt{(3 a+b)(3 b+a)})
$$

Where $a$ is the major and $b$ is the minor semi-axis.

Such approximation, is fairly easy to handle and it works well for the extreme case of $b=0$, a straight line, that tends to appear in the limit edge of the conoid and its boundaries.

For the said directrix, we can substitute $a$ for $R$, the radius of the circumference.

Thus, $a=R$ and it is easy to prove that, being $R / L$ the tangent of the angle formed by the middle section of the figure, the minor semi-axis is nothing but $b=x^{*} R / L$

As previously stated, $R$ is the radius of the end semicircle and $L$ is the total length and for any $x$ value between 0 and $L$, we would obtain,

$$
\mathrm{P}=\pi\left(3(R+x R / L)-\sqrt{\left(3 R+\frac{x R}{L}\right)\left(\frac{3 x R}{L}+R\right)}\right.
$$

And grouping similar terms,

$$
\begin{aligned}
& \mathrm{P}=\pi(3 \mathrm{R}(1+\mathrm{x} / \mathrm{L})-\sqrt{R(3+x / L) R(3 \mathrm{x} / \mathrm{L}+1)}) \\
& \mathrm{P}=\pi\left(3 \mathrm{R}(1+x / L)-\sqrt{R^{\wedge} 2(3+x / L)(3 \mathrm{x} / \mathrm{L}+1)}\right)
\end{aligned}
$$

The half perimeter is,

$$
\mathrm{P}_{1}=(\pi / 2)\left(3 R(1+x / L)-\sqrt{R^{\wedge} 2(3+x / L)(3 x / L+1)}\right)
$$


We can take $R$ out of the whole expression,

$$
\mathrm{P}_{1}=(\pi R / 2)(3(1+x / L)-\sqrt{(3+x / L)(3 x / L+1)})
$$

And $L$ as well,

$$
\mathrm{P}_{1}=(\pi R /(2 L))\left(3(L+x)-\sqrt{\left.3 x^{2}+10 L x+3 L^{2}\right)}\right)
$$

To obtain the lateral area of the conoid composed by diminishing strips along the central section, we need to perform the integration of,

$$
A_{c}=\frac{\pi R}{2 L} \int_{0}^{L}\left[3 L+3 x-\sqrt{\left.3 x^{2}+10 L x+3 L^{2}\right)}\right] d x
$$

The first two terms are immediate and give the solution;

$$
I_{1}+I_{2}=\left[3 L x+\frac{3 x^{2}}{2}\right]_{o}^{L}
$$

And the final result is for these two terms, applying the limits of integration is,

$$
I_{1}+I_{2}=3 * L^{2}+\frac{3 L^{2}}{2}=\frac{9 L^{2}}{2}
$$

This, multiplied by the constants out of the integral gives, $\frac{9 \pi R L}{4}$;

The third term is slightly more complicated being of the square root type and it involves a logarithmic primitive.

$$
I_{3}=\int_{0}^{L}\left[\sqrt{3 x^{2}+10 L x+3 L^{2}}\right] d x
$$

This integral presents the root of an expression of the type $a+b x+c x^{2}$.

As such, it is recommended to find the value of $\Delta=4 a c-b^{2}$, for this case, $36 L^{2}-100 L^{2}=-64 L^{2}$

The solution yields, [3]

$$
I_{3}=\left[\frac{6 x+10 L}{12} \sqrt{3 x^{2}+10 L x+3 L^{2}}-\frac{64 L^{2}}{24}\left(\frac{1}{\sqrt{3}}\right) \log \left(2 \sqrt{3\left(3 x^{2}+10 L x+3 L^{2}\right)}+6 x+10 L\right)\right]_{0}^{L}
$$

And substituting, 


$$
\begin{gathered}
I_{3}=\left[\frac{4 L}{3}(4 L)-\frac{64 L^{2}}{24}\left(\frac{1}{\sqrt{3}}\right) \log \left(2 \sqrt{3\left(16 L^{2}\right)}+16 L\right)\right]-\left[\frac{10 L}{12} \sqrt{3 L^{2}}-\frac{64 L^{2}}{24}\left(\frac{1}{\sqrt{3}}\right) \log \left(2 \sqrt{3\left(3 L^{2}\right)}+10 L\right)\right] \\
I_{3}=\left[\frac{16 L^{2}}{3}-\frac{5 \sqrt{3} L^{2}}{6}+\left(\frac{8 L^{2}}{3 \sqrt{3}}\right) \log (16 L)-\left(\frac{8 L^{2}}{3 \sqrt{3}}\right) \log (8 L \sqrt{(3)}+16 L)\right]
\end{gathered}
$$

By virtue of the properties of division of the logarithm,

$$
I_{3}=L^{2}\left[\frac{16}{3}-\frac{5 \sqrt{3}}{6}-\left(\frac{8}{3 \sqrt{3}}\right) \log \left(\frac{\sqrt{3}+2}{2}\right)\right]
$$

And from Eq. 12, the sum of the two previous immediate integrals was,

$$
I_{1}+I_{2}=\frac{9 L^{2}}{2}
$$

The total result for the so-conceived area, subtracting $I_{3}$ from Eq.17 is,

$$
\frac{\pi R}{2 L} L^{2}\left[\frac{9}{2}-\frac{16}{3}+\frac{5 \sqrt{3}}{6}+\left(\frac{8}{3 \sqrt{3}}\right) \log \left(\frac{\sqrt{3}+2}{2}\right)\right]
$$

After careful simplification, the Area the conoid based in $R$ and $L$ gives:

$$
I=\frac{\pi R L}{2}\left[\frac{27-32}{6}+\frac{5 \sqrt{3}}{6}+\left(\frac{8 \sqrt{3}}{9}\right) \log \left(\frac{\sqrt{3}+2}{2}\right)\right]
$$

Which is can be reduced to,

$$
I=A=\frac{\pi R L}{2}\left[\frac{5 \sqrt{3}}{6}-\frac{5}{6}+\left(\frac{8 \sqrt{3}}{9}\right) \log \left(\frac{\sqrt{3}+2}{2}\right)\right]
$$

And consequently,

$$
\mathrm{A}=\frac{\pi R L}{4}\left(\frac{1}{9}\left[15(\sqrt{3}-1)+16 \sqrt{3} \log \left(\frac{\sqrt{3}+2}{2}\right)\right]\right)
$$


For several reasons we have decide to name this new number $\psi$,

$$
\psi=\frac{1}{3^{2}}\left[15(\sqrt{3}-1)+\left(4^{2}\right) \sqrt{3} \log \left(1+\frac{\sqrt{3}}{2}\right)\right]=3.140923532703498
$$

$$
\psi \cong \pi
$$

For computational purposes both numbers can be equated and it will considerably simplify Equation 21 as,

$$
\mathrm{A}=\frac{\pi R L}{4}[\psi \cong \pi]=\frac{\pi^{2} R L}{4}
$$

We find such result remarkable in sundry senses. Firstly, because, $\psi$ is a transcendent number [4], akin to Pi but original in concept. Secondly, if substituted for Pi in the discussion it could perhaps improve the accuracy of Ramanujan's approach and this remains a question open for discussion in future developments.

Thirdly, it involves the fact that the area of the figure studied, the conoid, if $R=L=1$ would be a fourth of Pi squared or Pi multiplied by itself.

\subsection{Definition of the Antisphera}

A volume composed of four symmetrical opposed conoids as previously defined (radius=1) would have a lateral surface of $\pi^{2}$, that is Pi squared.

When conceiving such a form, obvious similarities with the sphere come to mind and that it is why we have named this curious figure in Grecian "Antisphera". The plan of the figure resembles a square of two by two, but the front view is a circle of diameter one. It constitutes a three-dimensional example of the circle's quadrature, sought since antiquity [5].

Its equation responds to,

$$
\frac{R^{2} z^{2}}{( \pm(R-x))^{2}}+y^{2}=R^{2}
$$

We consider just the former to be a noticeable finding. However, the problem is not completely solved since the parallel infinitesimal strips may not fit perfectly with the curved surface and they would act 
as a kind of envelope. For engineering purposes this is deemed sufficient when the angle of inclination of the figure, $\theta$, is not too steep (less than $\pi / 2.5$ ), but in order to improve accuracy we have calculated a coefficient to take into account the differences of width of the said strips.

Since we certainly know that at the middle section of the surface, the width of an actual unit section of the conoid is not $d x$ but instead $d x / \cos \theta$, we need to take into account this feature and effect an interpolation between both values.

It can be discussed if the variation of the strip is curvilinear or not, but several experimental calculations (See 2.4), estimate that for the time being, this is the preferred approximation for angles under pi/2.5 (other approaches are also being considered for higher values of $\theta$, see section 2.5).

Such coefficient of Cabeza-Lainez, $\kappa$, would accordingly be,

$$
\kappa=\frac{1+\sec \theta}{2}=\frac{1+\cos \theta}{2 \cos \theta}
$$

Where $\tan (\theta)=R / L$ as $\theta$ is the arctangent of the central section of the figure.

Therefore, the final expression to compute the lateral area of this particular surface, with the caveats referred above, produces,

$$
A=\frac{\pi^{2} R L}{4} \frac{(1+\cos \theta)}{2 \cos \theta}
$$

But if $\mathrm{R}$ and L coincide, Eq. 27 is reduced to,

$$
A=\frac{\pi^{2} R^{2}}{4} \frac{(1+\sqrt{2})}{2}
$$

In the case of a new volume that we have defined as Antisphera $\odot$, if $\mathrm{R}$ is the unit and if we adjoin the four quarters of which it is composed,

$$
A=\pi^{2} R^{2} \frac{(1+\sqrt{2})}{2}=\pi^{2} \frac{(1+\sqrt{2})}{2}
$$

Nevertheless, if we should make, 


$$
R^{2}=\frac{2}{(1+\sqrt{2})}
$$

It would mean that for an Antisphera of precise Radius $=0.9102$,

$$
A=\pi^{2}
$$

In this fashion, a new and distinct meaning, has been attributed to the second power of $\pi$, by virtue of such elaborate demonstration.

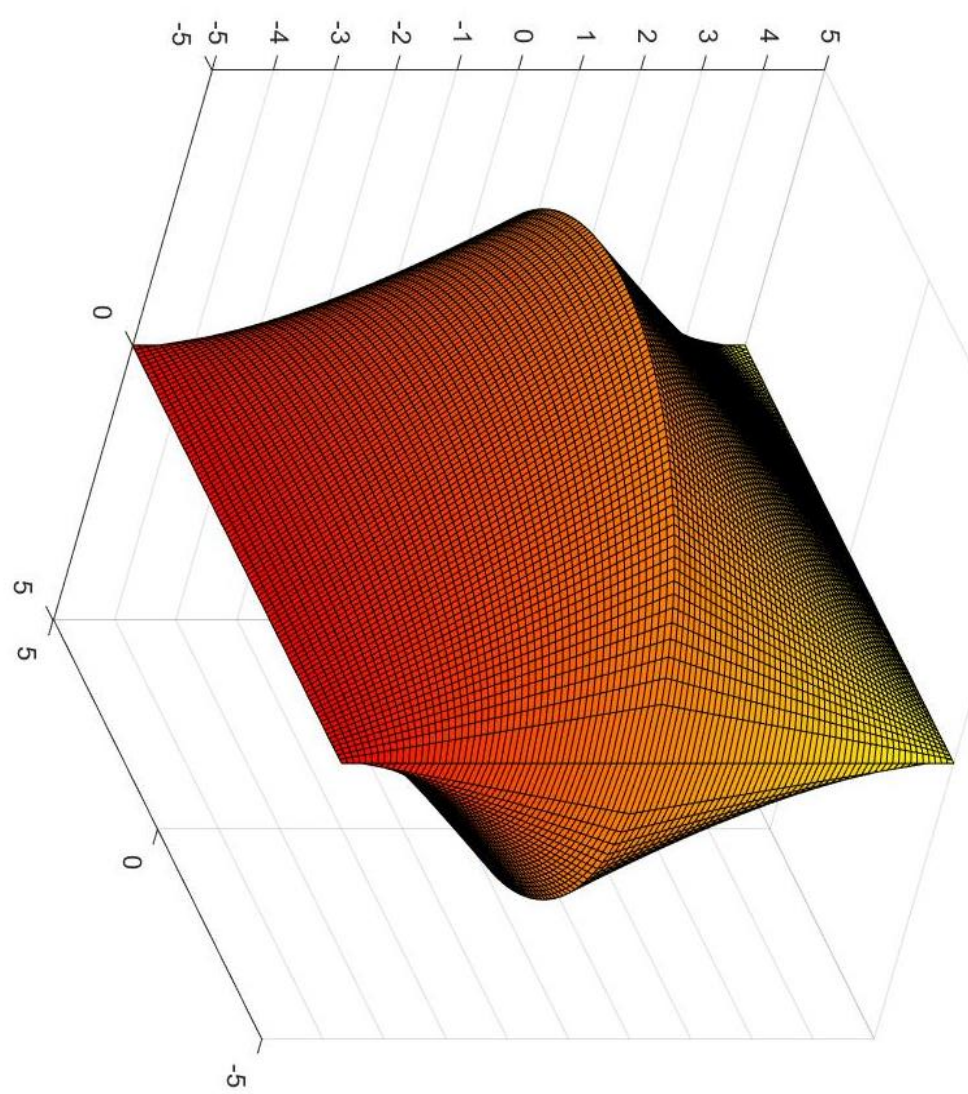

Figure 3. Depiction of the Antisphera for $R=5$.

\subsection{Comparison with other approximate computing methods}

Subsequently, we have proceeded to compere the aftermath with numerical simulation available, for instance the command Alphashape for computation of areas in Matlab and the graphic interface Grasshopper. The results show a considerable agreement and our findings stay in the middle of the output for both approximate tools (Table 1). 


\begin{tabular}{|c|c|c|c|}
\hline $\begin{array}{c}\text { Radius } \\
\text { = unit }\end{array}$ & $\begin{array}{c}\text { Alpha- } \\
\text { Shape }\end{array}$ & $\begin{array}{c}\text { Cabeza } \\
\text { Approx. }\end{array}$ & $\begin{array}{c}\text { Grass- } \\
\text { hopper }\end{array}$ \\
\hline 0.25 & 0.1958 & 0.1862 & 0.1842 \\
\hline 0.50 & 0.7712 & 0.7446 & 0.7369 \\
\hline 1 & 3.0726 & 2.9784 & 2.9476 \\
\hline 2 & 12.155 & 11.913 & 11.790 \\
\hline 3 & 27.734 & 26.805 & 26.528 \\
\hline 4 & 48.886 & 47.654 & 47.161 \\
\hline 5 & 76.019 & 74.460 & 73.690 \\
\hline 6 & 109.27 & 107.22 & 106.11 \\
\hline 7 & 148.83 & 145.94 & 144.43 \\
\hline 8 & 194.54 & 190.61 & 188.64 \\
\hline 9 & 246.41 & 241.25 & 238.75 \\
\hline 10 & 304.254 & 297.841 & 294.76 \\
\hline
\end{tabular}

Table 1. Results of computing the area of several conoids by the method proposed by author and with graphic interpolation procedures allowed by the software Grasshopper and the command Alphashape of Matlab.

More thorough data are being prepared, but the procedure for the graphic interfaces is clumsy and haphazard as a bespoke volume is required every time in order to compute the areas and the mesh of interpolation has to be decided beforehand with frequent hollow regions. This is a clear advantage of our method.

\subsection{Discussion for higher values of the conoid angle}

The $\kappa$ coefficient previously defined must, for coherence, remain over,

$$
\kappa>\frac{2 \tan \theta}{\pi}
$$

It is however clear that, for values of $\theta>1.25$ the former relation may cease to verify (Figure 4 ) 


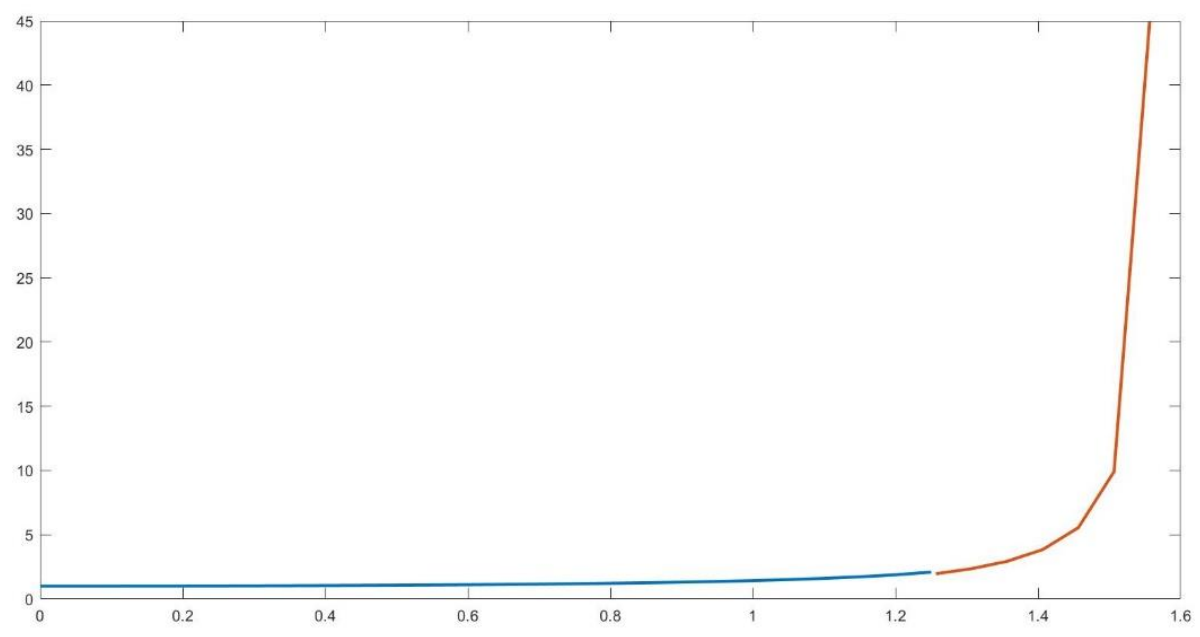

Figure 4. Depiction of Cabeza-Lainez coefficient $\kappa$ (blue) and limit case (red)

For lower quantities, the coefficient performs smoothly, since $\cos \theta$ tends to be one and the same does $\kappa$, so our prediction becomes even more accurate.

But for values over pi/2.5, it is convenient to define a new coefficient. We are currently estimating different adjustments to present them in a further development of our theory. In the meantime, a tentative assumption for the value of the area, would be the following,

$$
A=\frac{\pi^{2} R L}{4}+\frac{\pi R^{2}}{2}=\frac{\pi R}{4}(\pi L+2 R)=\frac{\pi^{2} R L}{4}\left(1+\frac{2 R}{\pi L}\right)
$$

Thus, the new coefficient $\kappa 1$ would be,

$$
\kappa_{1}=1+\frac{2 \operatorname{tg} \theta}{\pi}
$$

A suggested experimental refinement of this factor from $\theta=\pi / 2.5$ and onwards is,

$$
\kappa_{2}=\sin \left(\frac{\pi}{2}-\theta\right)+\frac{2 \operatorname{tg} \theta \sin \theta}{\pi}
$$

In most problems of engineering, high values of the said angle are rare because they imply that the conoid is very close to the circle or in other words, there is little or no space left inside the surface, which may become contradictory with the nature of spatial design [6]. 


\subsection{Calculations of the area for an elliptic conoid}

In 2.1 we had delayed the discussion after attaining the area values for the conoid ending in a circumference, but in a similar manner we can continue to use Ramanujan's prediction for an ordinate fragment of the same conoid whose extreme is logically an ellipse. For any real number $n$, the area yields,

$$
\begin{aligned}
A_{e c}= & \frac{\pi R}{2 L} \frac{(1+\cos \theta)}{2 \cos \theta} \int_{0}^{n L}\left[3 \mathrm{~L}+3 x-\sqrt{\left.3 x^{2}+10 \mathrm{~L} x+3 \mathrm{~L}^{2}\right)}\right] d x \\
A_{e c}= & \frac{\pi R L}{216} \frac{(1+\cos \theta)}{2 \cos \theta}[9(18 n(2+n)-(6 n+10) \sqrt{n(3 n+10)+3}+10 \sqrt{3})+ \\
& \left.96 \sqrt{3} \log \left(\frac{\sqrt{3 n(3 n+10)+9}+3 n+5}{8}\right)\right]
\end{aligned}
$$

For $\mathrm{n}=1$ the solution is Eq.21.

As before, we have employed the coefficient $\kappa$, but we need to be advised that for values of $\theta$ nearing $\pi / 2.5$ the expressions proposed in the previous section, that is, $\kappa_{1}$ or $\kappa_{2}$ should be introduced in its stead.

\subsection{Calculations of the volume of the conoid}

As a complement for the theories exposed, the computation of the volume of the conoidal figure by means of the previous integral method is relatively simple and exact. Since the value of the area of an ellipse is $\pi \mathrm{ab}$, and as before, $a=\mathrm{R}$ and $b=\mathrm{xR} / \mathrm{L}$

$V=\pi R \int_{0}^{n L}[x \mathrm{R} / \mathrm{L}] d x=\frac{\pi \mathrm{R}^{2} \mathrm{n}^{2} \mathrm{~L}}{2}$

This equates the volume of the equivalent cylinder multiplied by s=n/2.

If $\mathrm{n}=1$ and the limit is $\mathrm{L}, \mathrm{V}=\frac{\pi \mathrm{R}^{2} \mathrm{~L}}{2}$

that is half the volume of the equivalent cylinder (as $\left.\mathrm{s}^{\mathrm{1}}=1 / 2\right)$.

Finally, if $\mathrm{L}=\mathrm{R}$, the volume gives $\mathrm{V}=\frac{\pi \mathrm{R}^{3}}{2}$

making $\mathrm{R}=1$ as in the Antisphera we receive, $\mathrm{V}=\pi / 2$.

With $\mathrm{R}=0.9102$ as in Eq. 30, the volume reaches, $0.3770 \pi$ 
The volume and area properties in the conoid are smaller than in the cylinder but still larger than the equivalent cone. This will prove advantageous for the sustainability of structures and buildings (Sections 5 and 6) as the envelope and consequently the energy exchange and materials are less costly.

The finding of the volume of a conoid is sometimes attributed to Johannes Kepler but to our knowledge the first polymath to deduct it by comparison with the volume of a cone of the same basis, was Guarini in Euclides Adauctus[5].

\section{Repercussions for radiative heat transfer}

\subsection{Introduction to the problem of surface factors}

In previous researches [6], some of which were published in this journal [7], Cabeza-Lainez defined that the radiative exchange factor for any manifold surface is dependent on the equation:

$$
\mathrm{F}_{12}=\frac{1}{\mathrm{~A}_{1}}\left[\int_{\mathrm{A}_{2}} \int_{\mathrm{A}_{1}} \frac{\cos \theta_{1} \cos \theta_{2}}{\pi \mathrm{r}^{2}} \mathrm{dA}_{1} \mathrm{dA}_{2}\right]
$$

In a volume composed of only two surfaces, the same author established its second principle of radiation [8], which states that in the said situation, the rate of exchange is proportional to the areas of the intervening surfaces. For example, in a hemisphere (see Figure 4.), the respective areas are $A_{1}$ $=2 \pi R^{2}$ and $A_{2}=\pi R^{2}$.

As such, the corresponding form-factor from the half sphere to its base disc is [8],

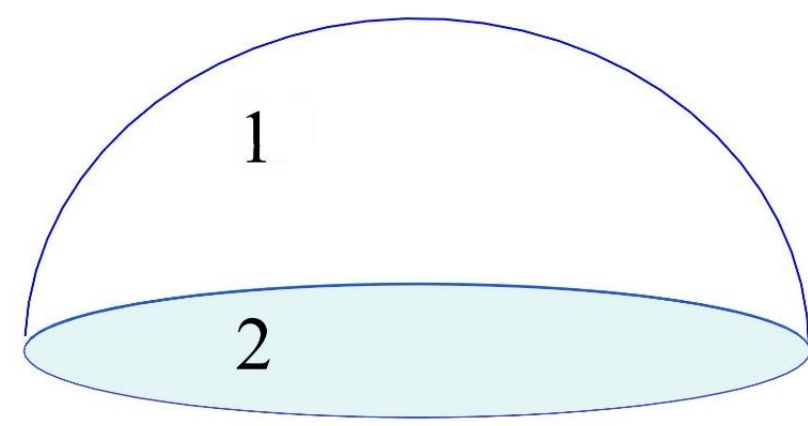

Figure 5. The second radiation principle of Cabeza-Lainez form-factors applied to a hemisphere

$\mathrm{F}_{12}=\frac{A_{2}}{A_{1}}=\frac{1}{2}$, and the amount of energy from the hemisphere to itself, $\mathrm{F}_{11}$ is also $1 / 2$ [8].

By the principle of conservation of energy all interchanges must add to $100 \%$ or the unity [6]. 
However, if the disc were topped by a double symmetric conoid, since previous to our finding, we ignored the value of its lateral area, the question remained unbeknownst.

We are finally in the position to respond to this issue with perfect ease. It is opportune to outline that the above integral equation (Eq. 41) is deemed unsolvable for the conoidal geometry. [7]

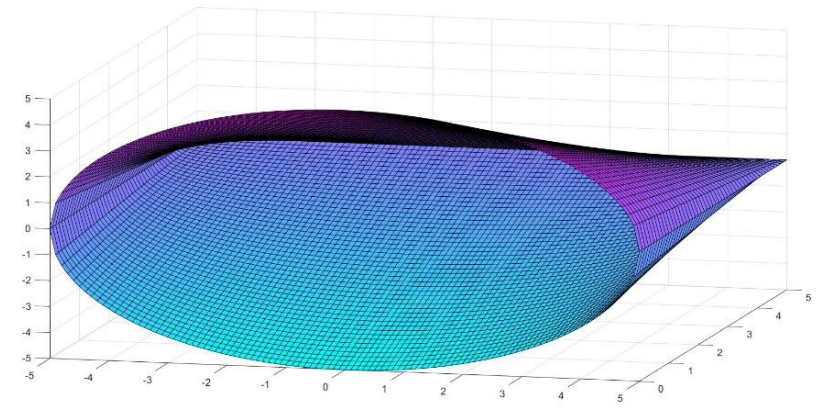

Figure 6. The second radiation principle of Cabeza-Lainez applied to the form-factor of a double conoid

The area of this double conoid is,

$$
A=\frac{\pi^{2} R^{2}}{2} \frac{(1+\sqrt{2})}{2}
$$

\subsection{Example 1}

In all the cases where $\theta=\pi / 4$, the relationship between the area of the base circle $\pi \mathrm{R}^{2}$ and the double conoid is precisely,

$$
F_{12}=\frac{A_{2}}{A_{1}}=\frac{4}{\pi(1+\sqrt{2})}=0.527393
$$

This is the factor from the conoidal top to the circular base and since $F_{11}+F_{12}=1$, the self-factor $F_{11}$ is then,

$$
F_{11}=1-F_{12}=1-0.527393=0.4726
$$

For this particular disposition, the values are not dissimilar from those of the hemisphere, 0.5, but they will need to be included in a standard comparison among radiative shapes described in reference [8].

Let us now discuss a new and more difficult situation for a conoid with radius 2 and length 4 . (Fig. 


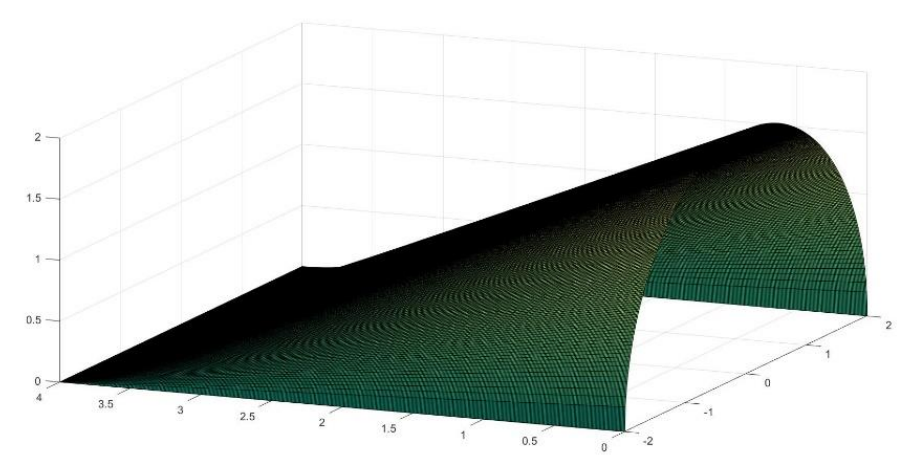

Figure 7. The single conoid limited by a rectangular plane and semicircle

\subsection{Example 2}

In reference [9], Cabeza-Lainez has partly solved Eq. 31 for some circular fragments.

For any vertical circular sector with a center situated in the middle of the edge $x$ of a horizontal rectangle of dimensions, $x$ and $y$; by virtue of the Cabeza-Lainez seventh principle, [10] the configuration factor from the sector of radius $r$ to a point in the perpendicular rectangle, will be:

Bearing in mind that the sector is comprised between the angles $\theta_{2}$ and $\theta_{1}$ and being its radius $r$ as mentioned.

$$
\begin{aligned}
& \text { For } \mathrm{t}=\mathrm{r}^{2}+\mathrm{y}^{2}+\mathrm{x}^{2} ; \mathrm{m}=\sqrt{x^{2} \sin ^{2} \theta_{1}+\mathrm{y}^{2}} ; \mathrm{n}=\sqrt{x^{2} \sin ^{2} \theta_{2}+\mathrm{y}^{2}} \\
& f_{12}=\frac{y}{2 \pi} \times\left(\frac{\cos \theta_{1}}{m} \arctan \frac{r}{m+\frac{\cos \theta_{1} x}{m}\left(\cos \theta_{1} x-r\right)}\right. \\
& \left.-\frac{\cos \theta_{2}}{n} \arctan \frac{r}{n+\frac{\cos \theta_{2} x}{n}\left(\cos \theta_{2} x-r\right)}\right) \\
& +\frac{y}{4 \pi x} \ln \left[\frac{\left(t-2 \cos \theta_{1} r x\right)}{\left(t-2 \cos \theta_{2} r x\right)}\right]
\end{aligned}
$$

In the usual situation of a semicircle the above expression is reduced to,

$$
f_{12}=\frac{1}{2 \pi}\left(\arctan \frac{r+x}{y}+\arctan \frac{r-x}{y}\right)+\frac{y}{4 \pi x}\left[\ln \left(r^{2}+y^{2}+x^{2}-2 r x\right)-\ln \left(r^{2}+y^{2}+x^{2}+2 r x\right)\right]
$$

By numerical procedures detailed in [7], we extend the above expression to the whole rectangle to find the form factor, whose value is of $F_{12}=0.1272$. It represents fraction of exchange of radiative energy from the horizontal rectangle under the conoid $\left(A_{1}\right)$ to the semi-circular side of the figure $\left(A_{2}\right)$. 
The previously unknown area of the conoidA3, following the above stated formulas (Eq.27) is 20.9042

From the reciprocity principle [6], $\mathrm{A}_{1} \mathrm{~F}_{12}=\mathrm{A}_{2} \mathrm{~F}_{21}$, whence,

$\mathrm{F}_{21}=(16 / 2 \pi) 0.1272=0.3239$

Being $\mathrm{A}_{1}$ and $\mathrm{A}_{2}$ planar, it is mandatory that [6],

$\mathrm{F}_{12}+\mathrm{F}_{13}=1$

$\mathrm{F}_{21}+\mathrm{F}_{23}=1$

And this implies,

$\mathrm{F}_{13}=1-0.1272=0.8728$

$\mathrm{F}_{23}=1-0.3239=0.6761$

Applying reciprocity again [6], $A_{3} F_{31}=A_{1} F_{13}$ and $A_{3} F_{32}=A_{2} F_{23}$, which yields,

$\mathrm{F}_{31}=\left(\mathrm{A}_{1} / \mathrm{A}_{3}\right) \mathrm{F}_{13}=(16 / 20.9042) 0.8728=0.6680$

$\mathrm{F}_{32}=\left(\mathrm{A}_{2} / \mathrm{A}_{3}\right) \mathrm{F}_{23}=(2 \pi / 20.9042) 0.6761=0.2032(53)$

By virtue of the principle of conservation of energy [6],

$\mathrm{F}_{31}+\mathrm{F}_{32}+\mathrm{F}_{33}=1$

$\mathrm{F}_{33}=1-\mathrm{F}_{31}-\mathrm{F}_{32}$

Being non planar, the fraction of energy that the radiating conoid exchanges with itself is,

$\mathrm{F}_{33}=1-0.6680-0.2032=0.1288$

Not merely radiative heat transfer in the figure under study has been solved by this procedure, but also light transmission when it originates at conoidal skylights like those constructed by Ilja Doganoff [11] in 1957 in Bulgaria (Figs. 19 and 20).

\subsection{Example 3}

If we, as in a sort of check, would double the conoid presented in Ex. 2 and compare in it the factor between a circle $\left(A_{2}\right)$ and $\left(A_{1}\right)$ the enclosing figure, (Fig. 8) we would obtain that, since the relation of areas is now 0.3006 , the factor from the conoid to the circle is precisely this value, following CabezaLainez' second principle [8].

Accordingly, the complex self-factor of the conoid to itself yields nothing but, 
$F_{11}=1-0.3006=0.6994$

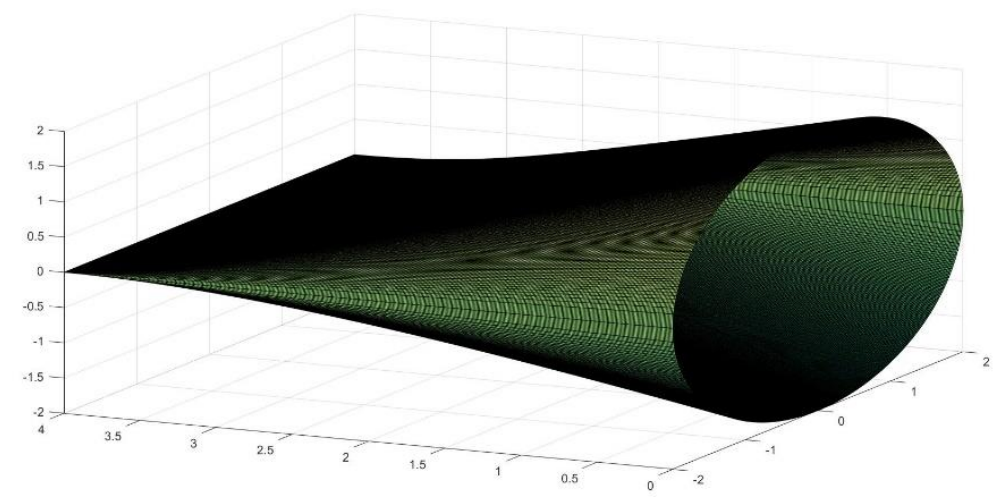

Figure 8 . The same conoid of figure 7 but closed to obtain the form factor

In the original half Antisphera, if we remember 3.2, and Ex.1, the same quantity amounted to,

$\mathrm{F}_{11}=1-0.527393=0.4726 \quad(58)$

Such difference can be explained because the area of this new conoid is larger than the previous one since the angle $\theta$ is less pronounced (in this case, the length doubles the radius), or in other words more energy is retained under the new configuration.

In the second example discussed of a half-conoid (3.3), involving three surfaces (rectangle, semicircle and conoid), the self-factor was smaller, 0.1288 , but a noticeable fact is that even so, it does not reach a fraction of one half of the self-factor for the whole conoid $(0.6994 / 2=0.3497)$ of the same dimensions (Ex. 3), as logic would perhaps induce to think. This exemplifies the complexity of the solutions because they often appear to rule out common sense [12].

It is important to stress the utmost difficulty of obtaining these entities by any other method, including quadruple integration [7]. 


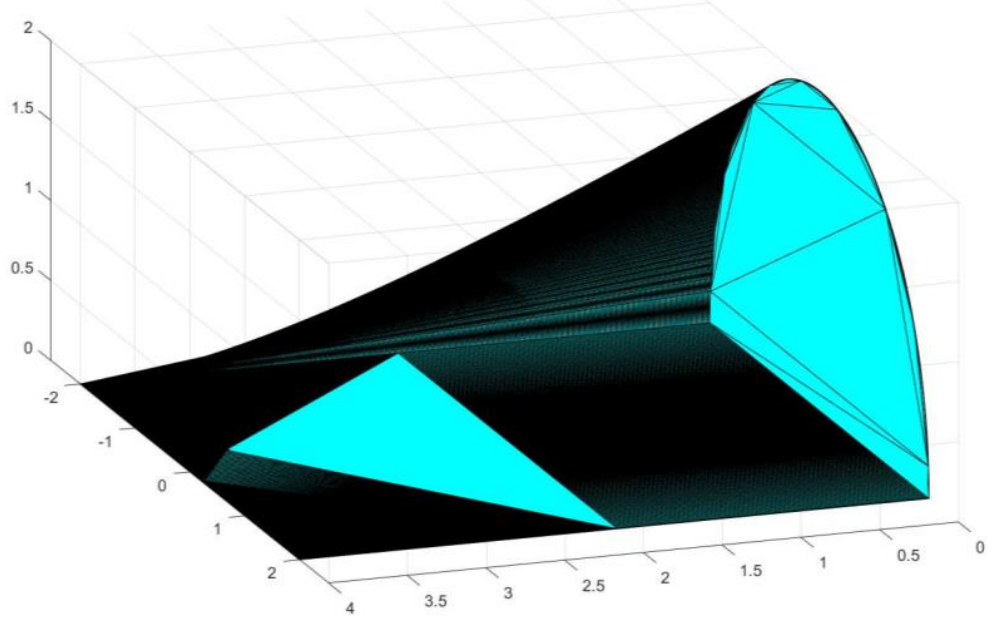

Figure 9. The three surfaces intervening in Example 2 whose area is calculated by computer graphic interpolation

\section{Generation of new figures based on the previous findings}

In Section 2, we defined the symmetrical figure composed of four conoids, namely the Antisphera. [Fig. 10]

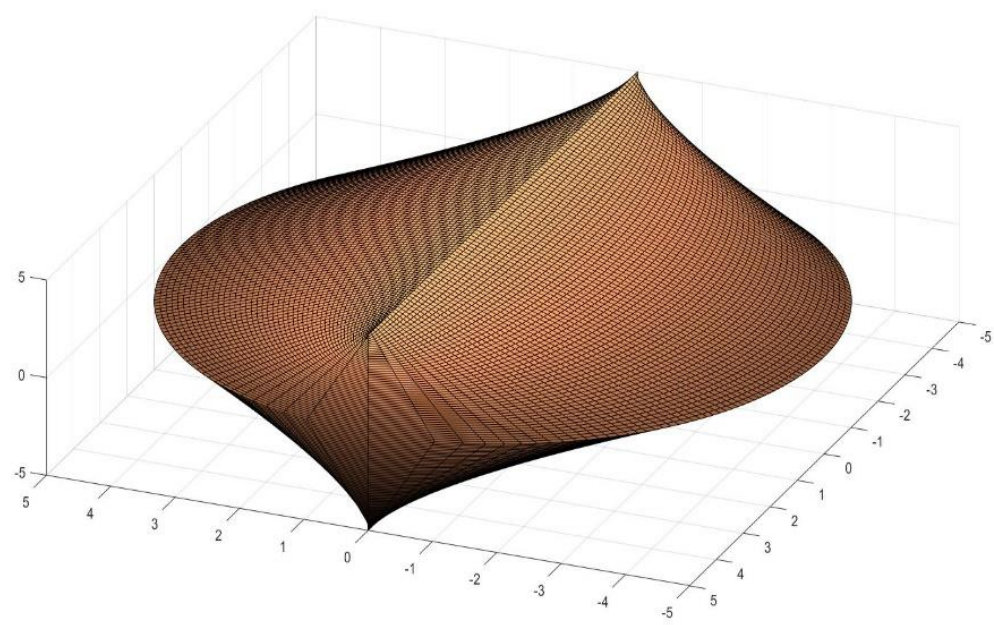

Figure 10. Three-dimensional rendering of the Antisphera

Nevertheless, we have conceived that by altering the symmetry and parts of the previous figure, a series of other interesting bodies derives, maintaining initially the ratio $\mathrm{R}=\mathrm{L}$. 
The first one is opposed geometrically to the Antisphera because the edge straight lines intersect at its center plane, which is void. Due to this elusive and somewhat dual nature we have coined the name Dyosphera@ for this shape [Fig. 11].

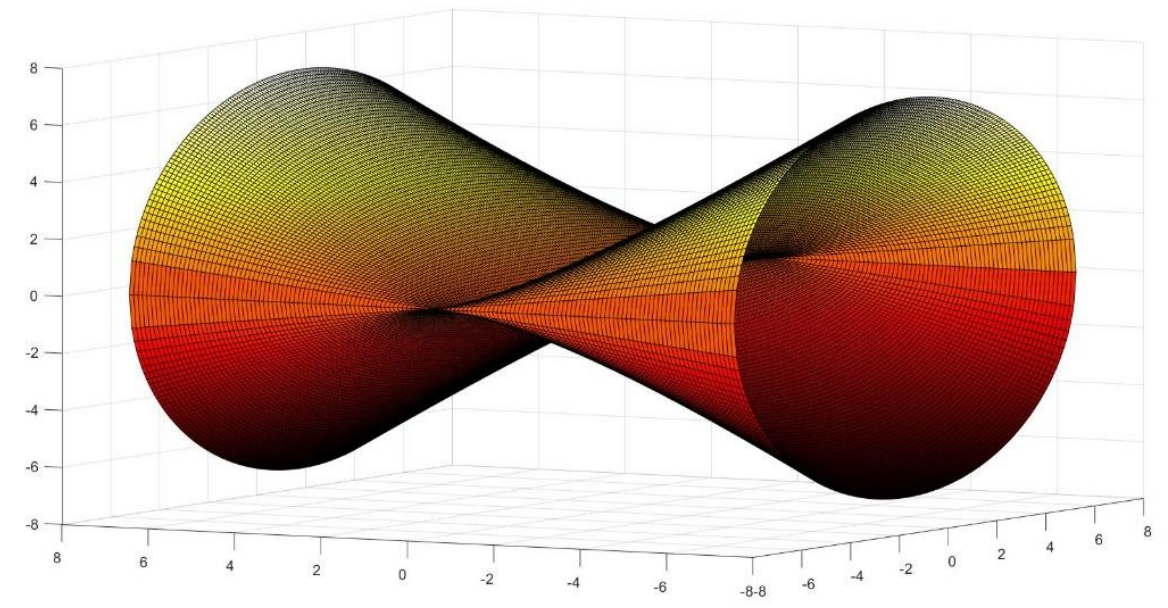

Figure 11. The horizontal part of the body known as Dyosphera

In total, the Dyosphera features eight conoidal sections, organized in groups of four, rotated $\pi / 2$ degrees [Fig.12]

The corresponding Equations are,

$$
\frac{R^{2} z^{2}}{x^{2}}+y^{2}=R^{2}
$$

Combined with,

$$
\frac{R^{2} x^{2}}{z^{2}}+y^{2}=R^{2}
$$

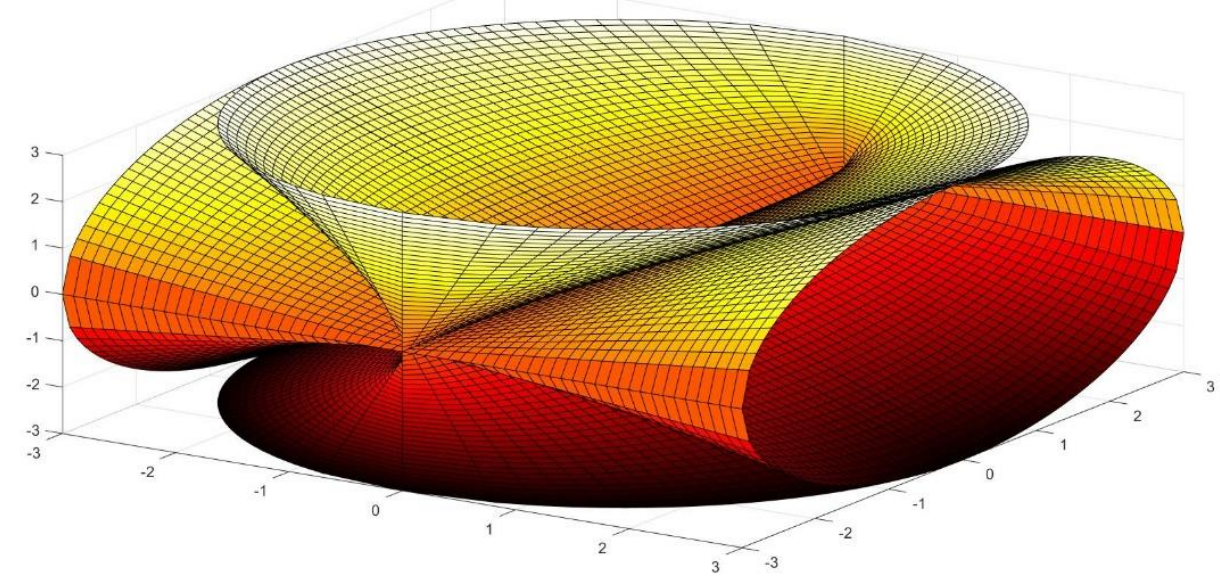

Figure 12. Distorted depiction of the complete Dyosphera. 
The open cavities and sinuous receptacles of this figure make it particularly suitable for aeronautical and machinery parts. It is also very stable form because it possesses four circular bases.

Horizontal parts of the Dyosphera, can be adroitly combined with the upper part of the Antisphera [Fig. 13], to obtain a different figure that we have called Alosphera@.

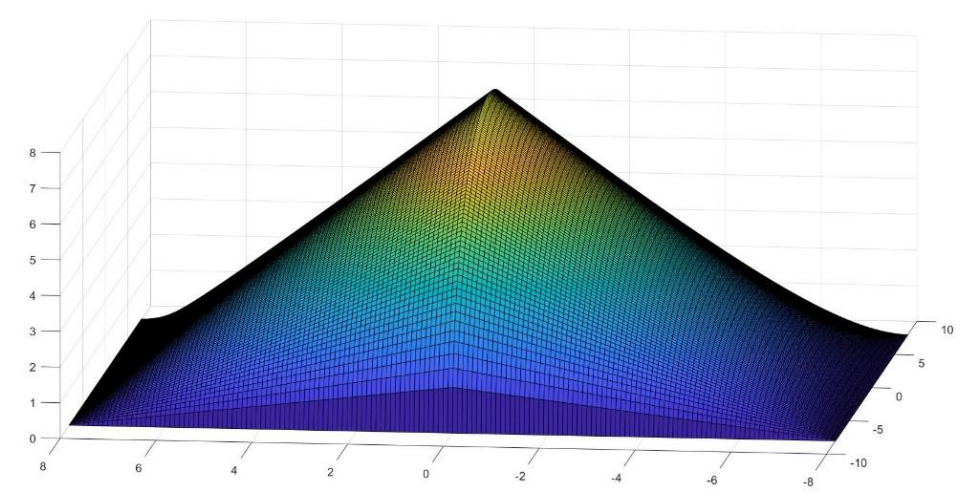

Figure 13. Detail of the upper part of the Antisphera

In fig. 14, we can see represented the Alosphera, its main feature is that two bases are square and two circular, what makes it suitable por different uses, with straightforward storage of various units and we have come to guess that some cellular growths of different organisms may respond to this evolutive pattern.[13]

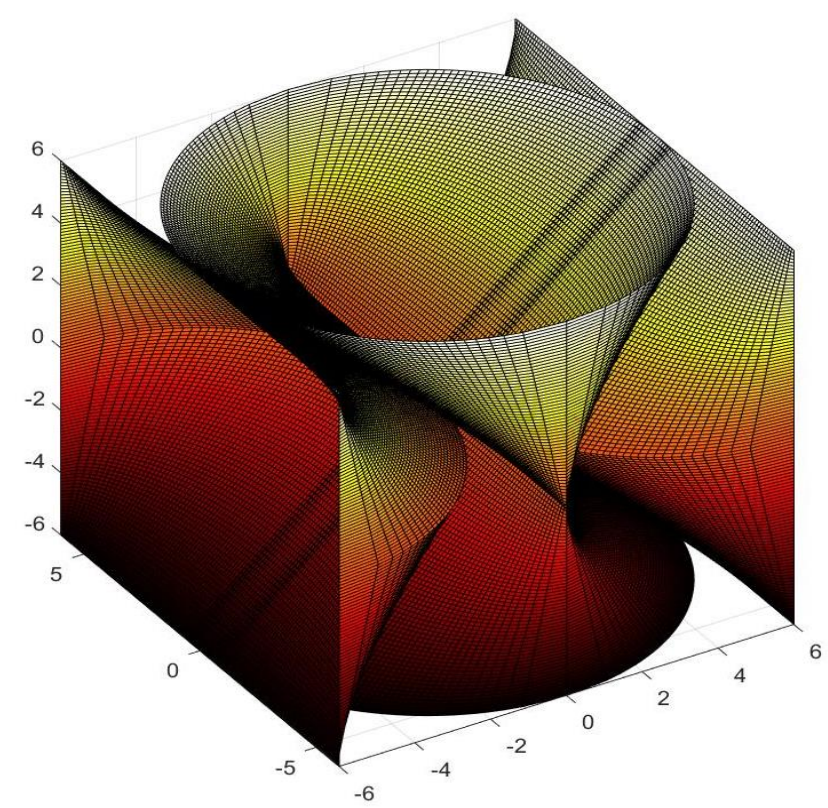

Figure 14. The Alopshera.

The Alosphera is the first Antisymmetric figure that we have identified but, like the Dyosphera, it also presents eight conoids. 
Finally, a very important antisymmetric finding is the Pterasphera [Fig. 15].

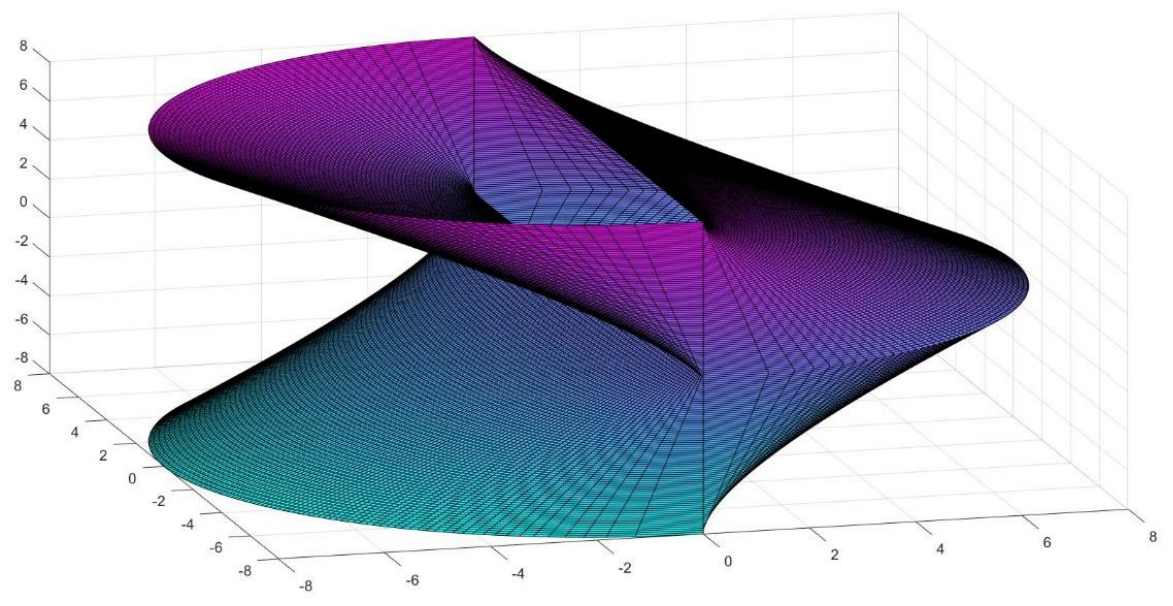

Figure 15. Initial Section of the Pterasphera

This body as we shall discuss in section 6 is tubular in nature. Being internally connected in its entirety, it is apt for conducting all kinds of fluids in an advantageous manner since for instance it can reduce the velocity and at the same time the noise of transporting the required fluids. Unlike Antisphera it is self-standing and well balanced, which turns it suitable for elongation in the manner of a tower.

\section{Architectural and Engineering Significance of the geometric findings}

\subsection{Historical Evolution}

To our knowledge the first mathematician to introduce the conoidal figure was C. Guarino Guarini [5] in his famous treatise Euclides Adauctus et Methodicus.
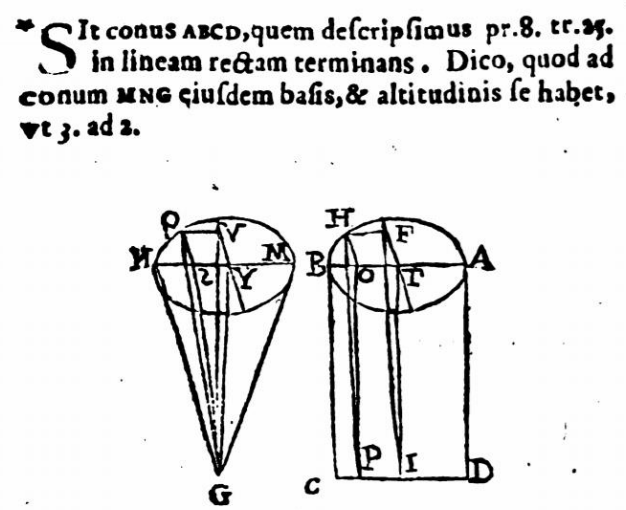

Figure 16. The point-cone and the cone that ends in a line according to Guarini 
He claims that only he has discovered the form and says that it is a cone that ends in a straight line. (Later he uses the word hyperbolic conoid but for an entirely different body [5]).

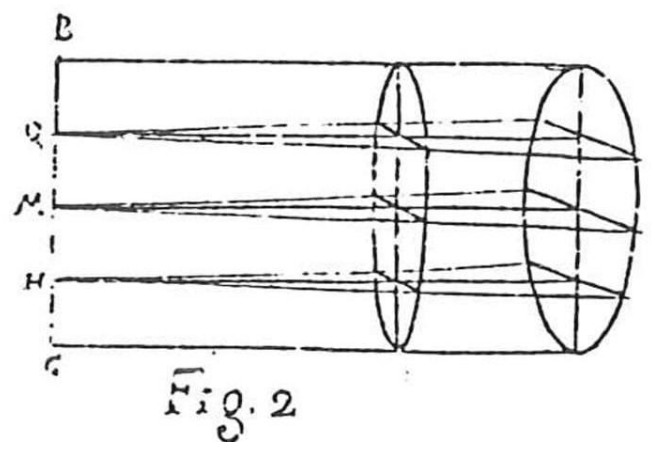

Figure 17. Architettura Civile. Camillo G. Guarini. Lastra IX. Trat. IV. Depiction of a cone ending in a line

In another monumental work entitled Architettura Civile, published posthumously in 1737, again he refers to this unusual cone, stating that it has limited applicability in the corners of chamber vaults we surmise that as a kind of squinch. He goes so far as to calculate the volume of the figure in the sense that we have explained in section 2.7 .

After this, the form remains all but forgotten in Architecture until, at the beginning of the $20^{\text {th }}$ century it receives a definitive impulse by the hand of revival architects like Antonio Gaudi, who designed a roof with serially alternated conoids for a school building in front of his Sagrada Familia Cathedral in Barcelona (Fig. 18).

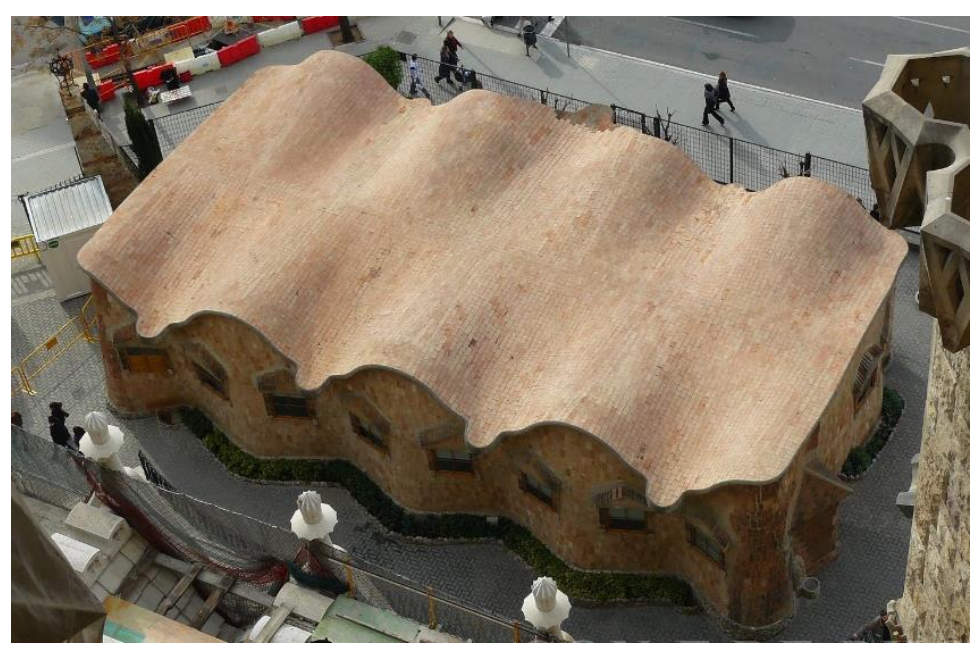

Figure 18. The Sagrada Familia Schools re-built. A. Gaudi 
Around the 1930s, the advent of concrete Shell construction favored a revolution of engineering forms and the conoid surface was recipient of much interest especially for building hangars, factories and warehouses [14].

One of the best extant examples is the work of the Bulgarian Engineer Ilja Doganoff who, in 1956-57 erected a repair workshop for Bulgarian Railways featuring a hundred conoid skylights

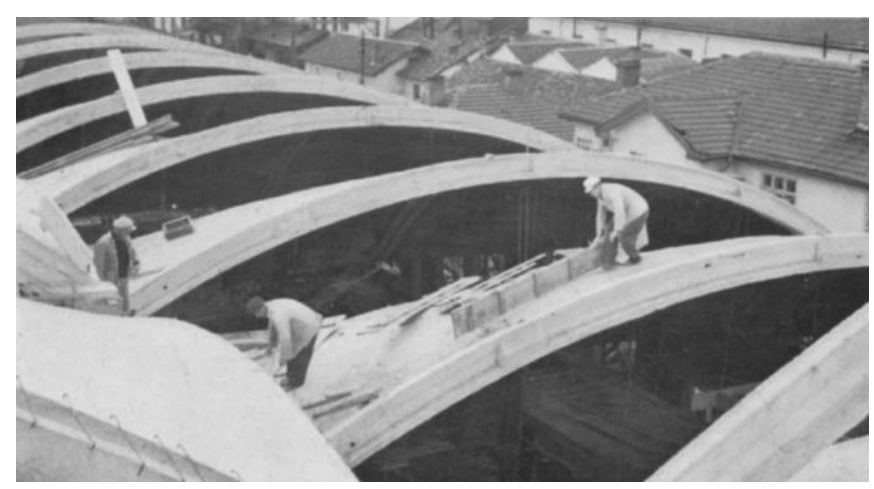

Figure 19. Ilja Doganoff. Construction works for the Railway Depot (1957).

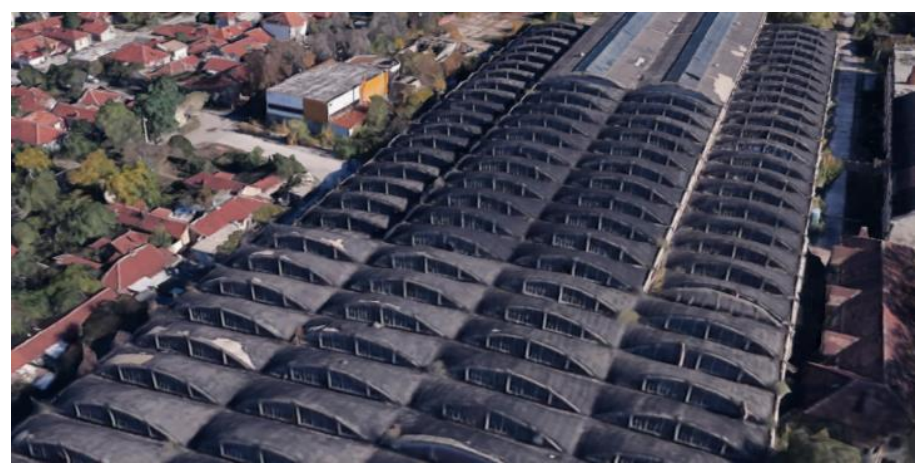

Figure 20. Aerial recent view for the Railway Depot by I. Doganoff.

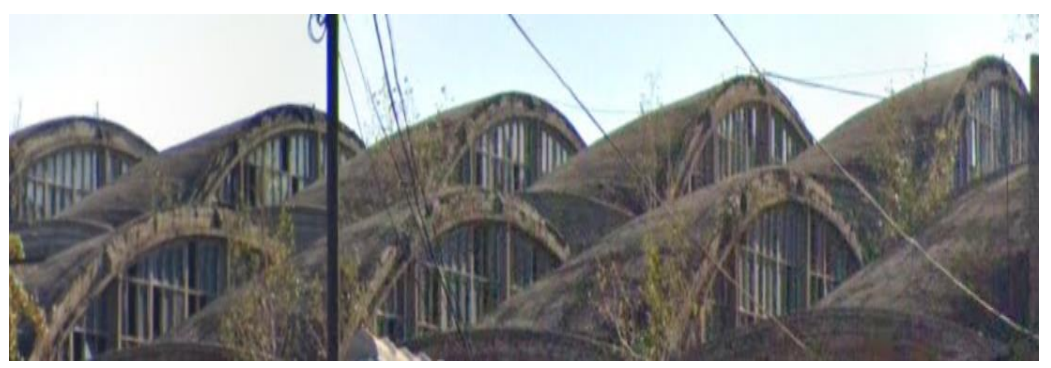

Figure 21. Close-up of the current state of the conoids still standing after 64 years without maintenance

The shapes were prefabricated in situ and then put on the roof with the help of a crane. They are an example of the extreme feasibility of the surfaces. We have calculated the daylighting transmission of similar shapes in section 3 what neither Doganoff nor Ramaswamy [14] where able to perform. 
Ramasamy[14] and Doganoff $[11,15]$ report that owing to the want of knowledge about the surface, structural calculations turn out to be cumbersome but still engineers cherish the form because of its many advantageous properties and elegance, citing lighting and economy of construction as potential reasons to explain their predilection.

\subsection{Recent Projects of conoids realized by J. M Cabeza Lainez}

The present author has been working in such conoid shapes for more than twenty-five years and his experience has ignited in part the present article. Modelling of the characteristic structural, acoustic and lighting properties of conoids encompasses a significant amount of my career as researcher. Based on that, I can attest to its sustainability and endurance [16].

From the structural point of view, as a ruled surface it can be built directly through straight lines (beams or poles), this fact greatly facilitates the construction and scaffolding, as more natural materials like bricks or bamboo rods can be used without difficulty, even for reinforcement or repair. Carbon-fiber coating has become a recent alternative for reinforcement although moderately expensive.

The arched section of the conoid whether circular or elliptic presents a vertical tangent as we have shown in the previous sections [16]. Therefore, if adequately constructed it is free from horizontal thrusts which might compromise the supporting frame. In other words, it transmits all the loads of the structure vertically and avoids the use of buttresses (Fig. 22).

These consistent and diminishing arches function as girdles for most part of the surface and provide increased resistance to a significant amount. It is true that calculation of hyper-static arches is not widely treated in the literature but we suggest the column analogy method proposed by $\mathrm{H}$. Cross [17] as a helpful and programming-friendly procedure.

Due to its curvature the aerodynamics of the roof is excellent for bearing wind loads and other meteorological phenomena as rain, drizzle or snow. At the same time, because of the former it enhances air flow from the outside or from internal stack effect with appropriate vents.

Regarding lighting properties if, as usual, the glazed apertures lie in the curvilinear extremes of the forms, they bring uniform illuminance as we calculated in section 3 (Figure 23) and can be easily shaded by eaves protruding from the same brim of the surface (Figure 24)

Acoustic properties stem from the circumstance that the inside surface of the conoid is mostly convex as we have checked mathematically [6]. Sound waves are diffused in this kind of ceiling and consequently, noise and reverberation become dampened. If, through appropriate design, the conoid covers a trapeze or fan-shaped plan (Figs. 25,26) the effect of an even sound pressure is manifest [18]. 
(In this last case the surface is not a proper conoid as the forming lines are not all parallel to a common plane).

The aforementioned acoustic benefits are extensive to interior illumination for the same reason of convexity of forms.

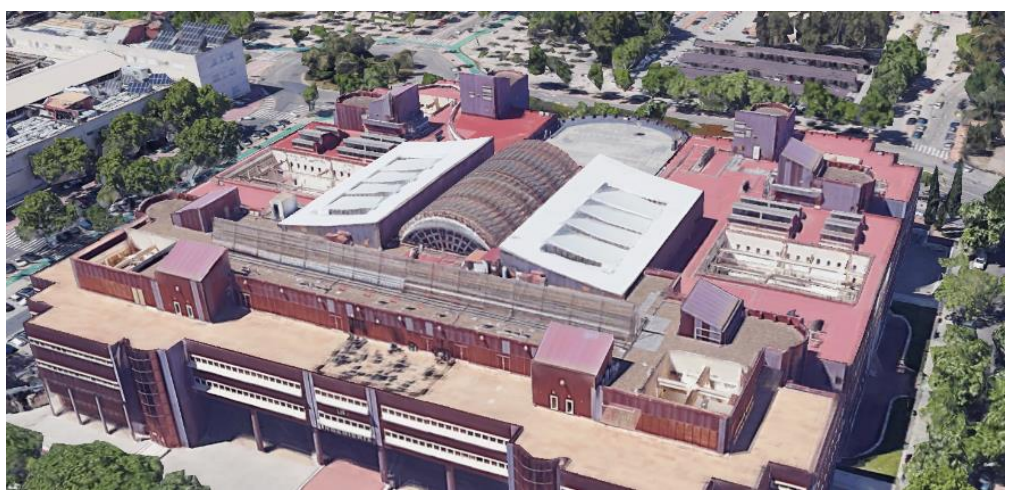

Figure 22. School of Engineering of the University of Sevilla. Aerial view of Conoidal Skylights. Arch. J. L. Asiain and J. M. Cabeza

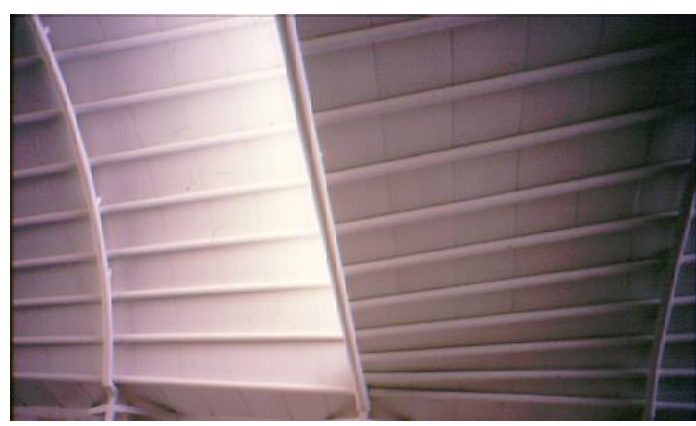

Figure 23. Interior view showing light diffusion at the central conoid. School of engineering of Seville.

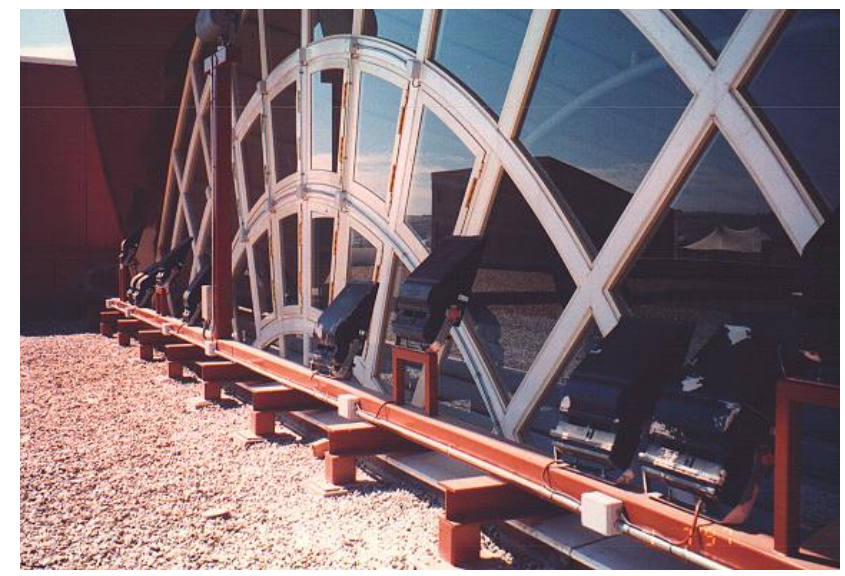

Figure 24. Semicircular opening and projecting overhang at the extreme of the central conoid of the School of Engineering of Seville. 


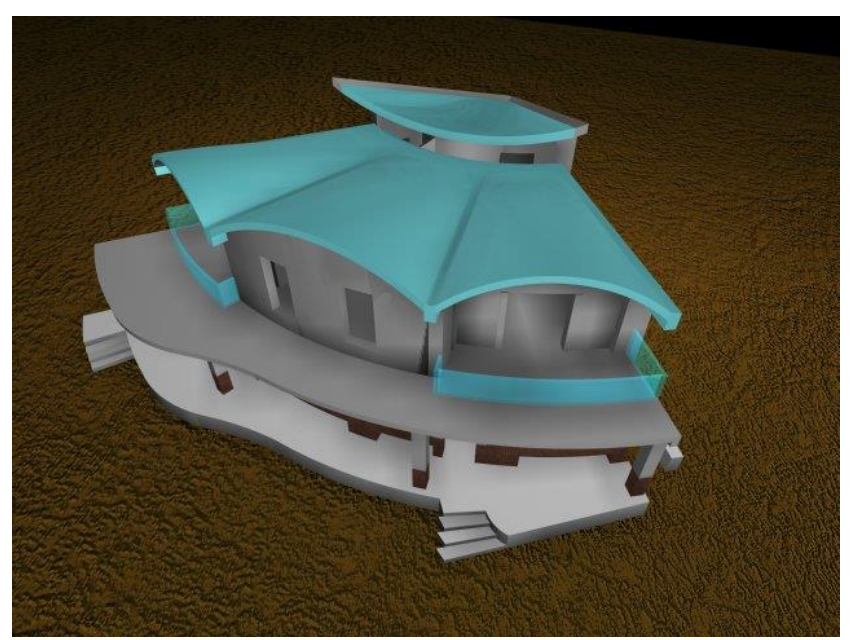

Figure 25. Three rotated conoidal roofs designed for a musicians' family. Sanlucar (Seville). Arch. J. M. Cabeza Lainez

Although strictly-speaking not a conoid, because its equation differs from what we have explained above, the cover of a fan shaped plan with this kind of surface offers a very interesting structural property: the larger spans between pillars are covered by arches while the tapered end of the trapeze features a common slab or planar beam, what seems very logical from the constructive aspect [18].

In this way, the shells' materials can be lighter and smoother, in fig. 27 we present three vaults consisting of thin layers of hollow brick with steel mesh as a reinforcement. The result has proven to provide increased insulation and adds variety of light effects (Fig.28).

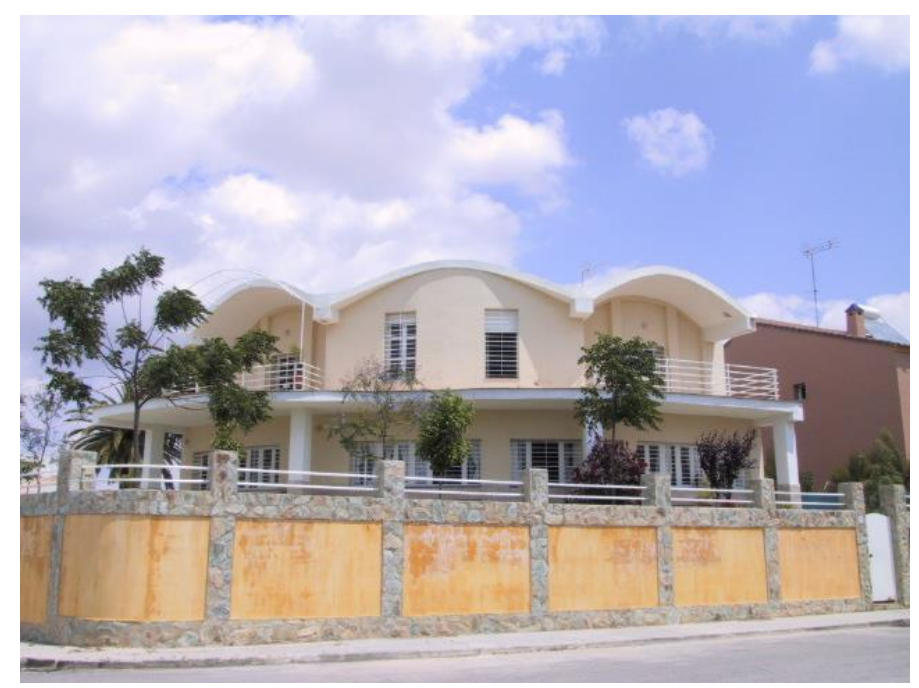

Figure 26. View of the house of the musicians. The vaults are entirely constructed in brick with occasional steel reinforcement. 


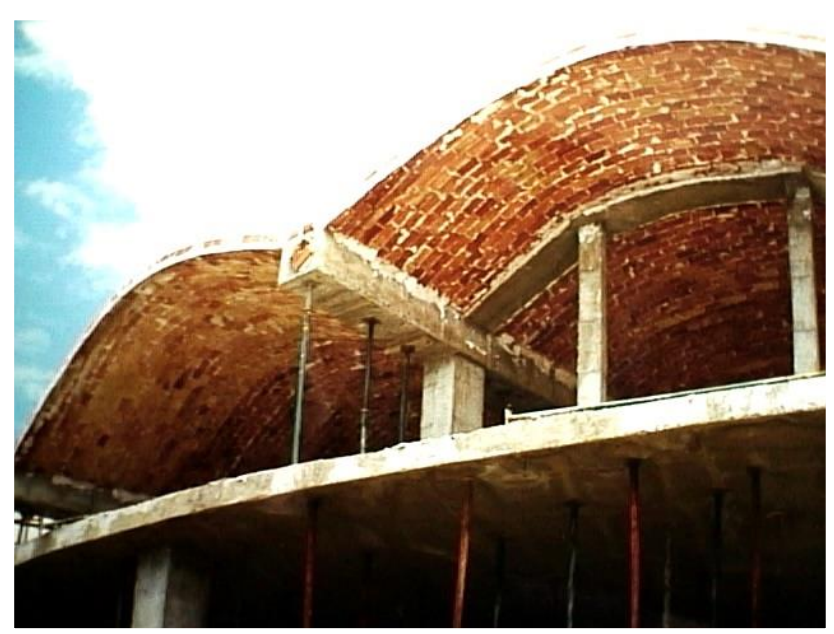

Figure 27. Vault pieces are constructed in hollow thin brick with steel mesh for reinforcement and attached to a concrete frame.

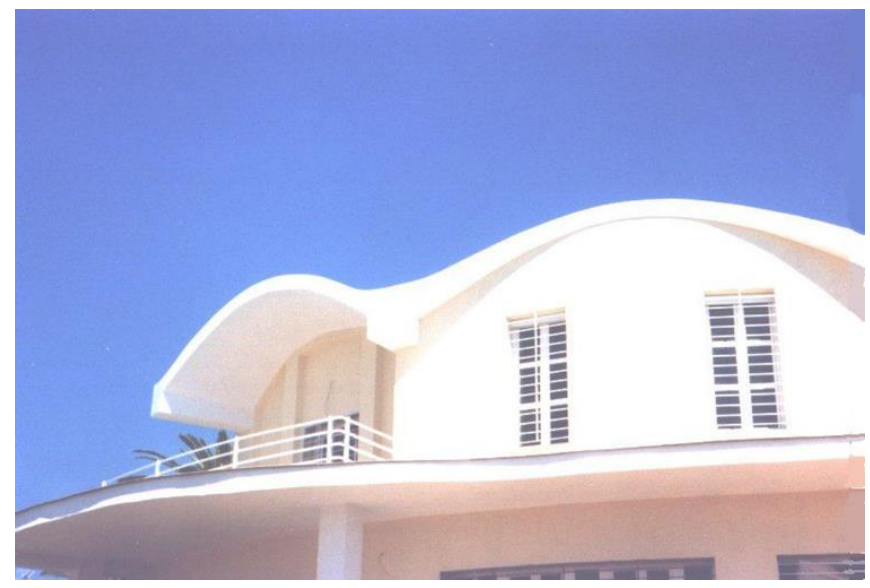

Figure 28. Detail of pseudo-conoid roofs in which the sun-path produces intriguing variations through the day

\subsection{Future Proposals}

As a corollary for the theories exposed we will discuss two project-forms that we have created with conoids taking into account evolving technologies. The firs one is a system of skylights similar to the one depicted in Figs. 21 and 24, but in this case, the glazed parts instead of being planar are also conoids (Fig. 29). Bearing in mind the discussion on heat and light transfer of Section 3, this feature presents undoubted advantages [19]. Firstly, the glazing is better shaded and protected by the opaque upper conoid. Secondly, sunlight and heat transmission are modulated by the smooth curves conjoined to the innovative glass properties. In this way the glass surface becomes load-bearing and collaborates with the general structure. 


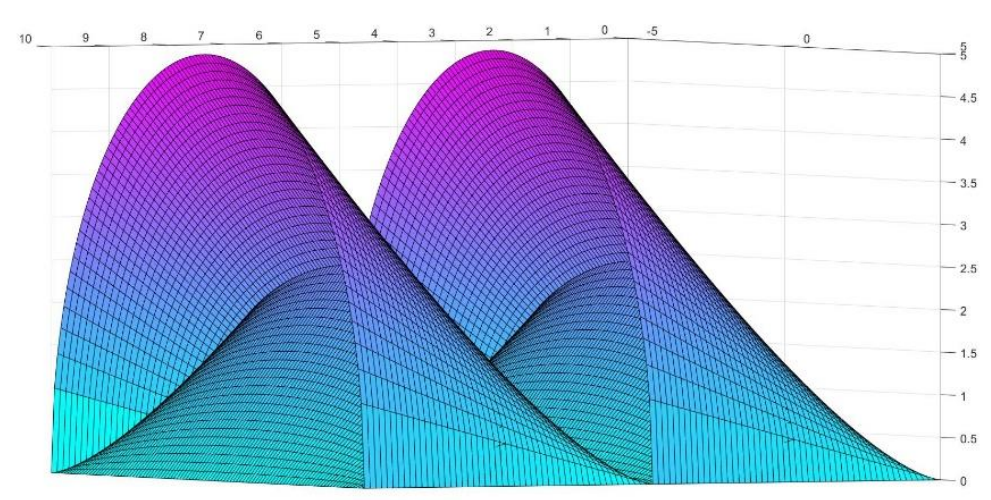

Figure 29. Outline of proposed Skylights with internal conoid glazing

The new skylights are more impervious, break-proof safer and cleaner in the absence of maintenance, as dust collection is diminished with the curvature.

The second form consists of an innovative proposal for an amphitheater, music or sports venue (Fig. $30)$.

In this case, being a double conoid, the advantages previously exposed are improved. The sections are closed curves, like the ellipse and the circumference in the brim. Thus, they work as tension rings or girdles to hold the structure together without severe deformations. The bearing capacity of the shape is extreme. The tiers of the amphitheater are the obverse of the external façade, there is no need to superimpose a conical structure inside a cylinder like in the Colosseum or in Spanish bullrings. Among other problems, the ancient structures forced to build giant discharge vaults and galleries which transferred severe thrusts to the outer façade. As a result, we have calculated that savings in building materials of this proposed facility could be massive.

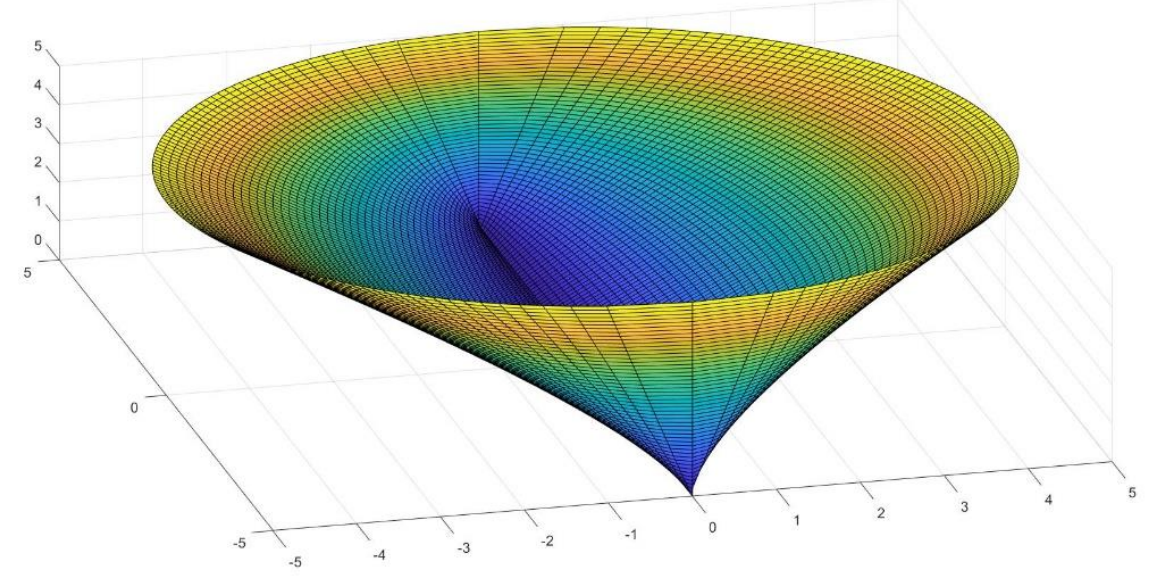

Figure 30. 3-D drawing of the new Amphitheater. 
Still, the structure can be easily constructed with straight beam elements and reinforcements. The foundations are pointing to the soil as in a kind of arrow, that means that it will be very stable, safe and simple to develop.

The outer surface of the conoid is not vertical but inclined and so the surroundings of the amphitheater would be self-shaded, an interesting feature in warm climates.

As for the grandstands, it is not difficult to adapt awnings or other shading systems to the inside area in order to protect the tiers from the rain or the sun (Fig.31)

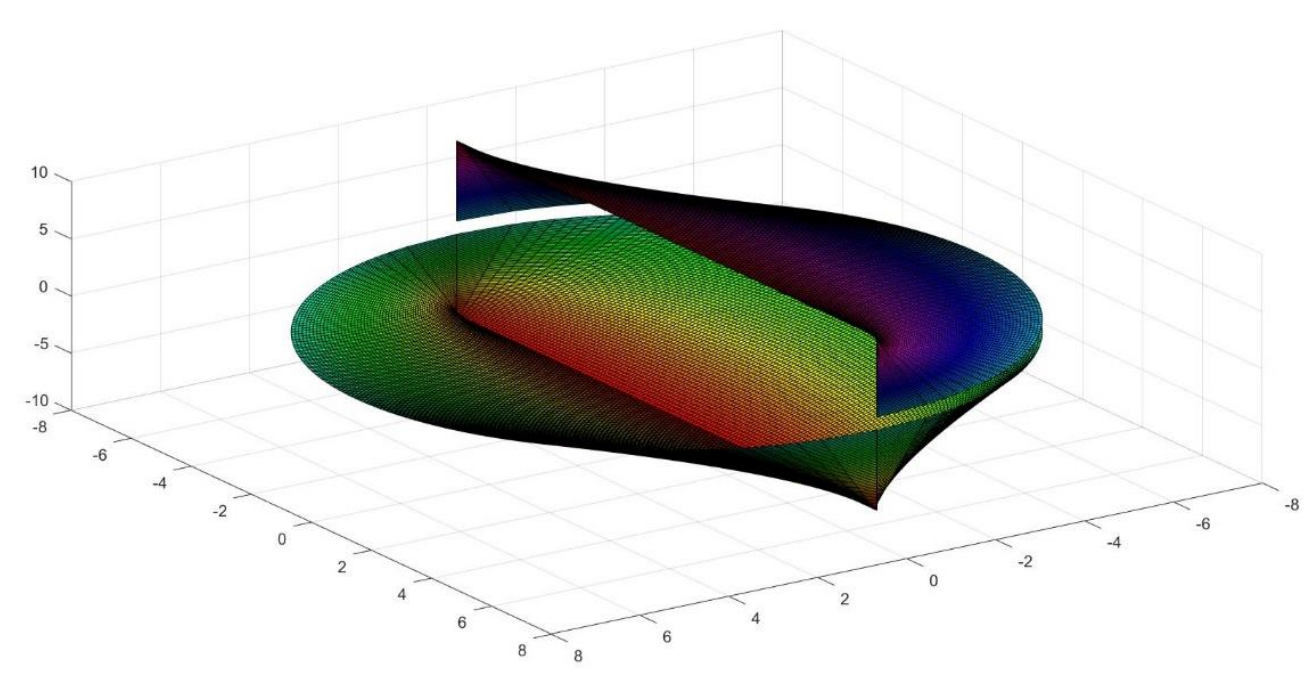

Figure 31. The Amphiteater with proposed retractable shade

As mentioned above, the effect of concentration of sound, rather annoying in conventional stadiums will be almost completely avoided due to the convexity of the surface. In reference 6 we have demonstrated this by acoustic ray-tracing procedures. These rely on the finding of the normal to the conoid surface at each point.

The procedure to extract the normal is first order differentiation of the equation of the surface defined as $F(x, y, z)$. The normal vector is obtained as $N=\left(F_{x}, F_{y}, F_{z}\right)$ and in this case, has the value of:

$$
N=\left(\frac{R^{2} z^{2}}{(R-x)^{3}}, y, \frac{R^{2} z}{(R-x)^{2}}\right)
$$

We can trace a vector field with the reflected sound-rays from an emission point to check that they are effectively dispersed in the air and not concentrated [6]. 
The only remaining questions would be those of selecting the relative heights of the stages and platforms and other design issues like circulation in the venue. Nonetheless we believe that our proposal could be another good example of how the conoid-based bodies are able to create a significant volumetric space with a comparatively small enveloping surface, the key is that they offer a high spatial compactness which is usually an added value in terms of heat exchange, costs reduction and sustainability in general.

\section{Repercussions for technology}

So far, we have presented scientific design developments that we hope will find a myriad of applications in technological areas like Nautical, Aerospace, Building, Heritage, Retrofit and associated industries or machinery. Such fact attests to the versatility and feasibility of the solutions presented which derive from our mathematical investigation. In the last part of the research, due to their complexity, the surfaces have been materialized with the help of 3D fabrication procedures to decide on some difficult points of the equations or either on future realizations of the proposals. It is undeniable for us that the results attain the domains of Art and Design (Figs. 32-35). This will perhaps merit a separate study.

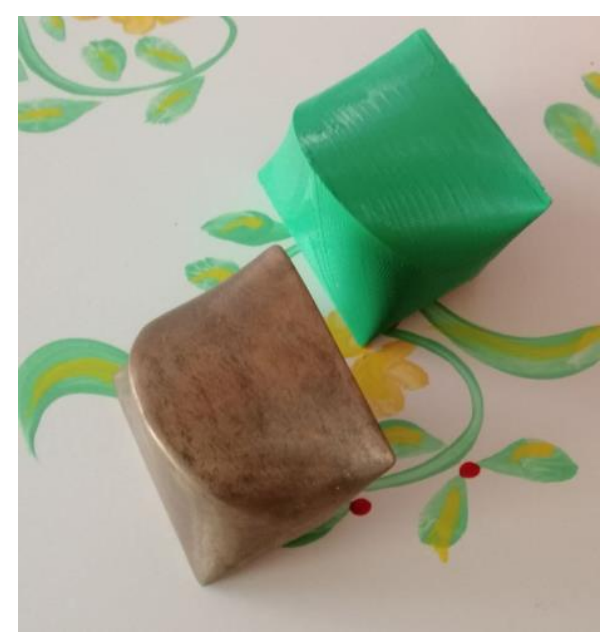

Figure 32. Antishpera in bronze by the artist Sergio Portela and in plastic 3D print (green). 


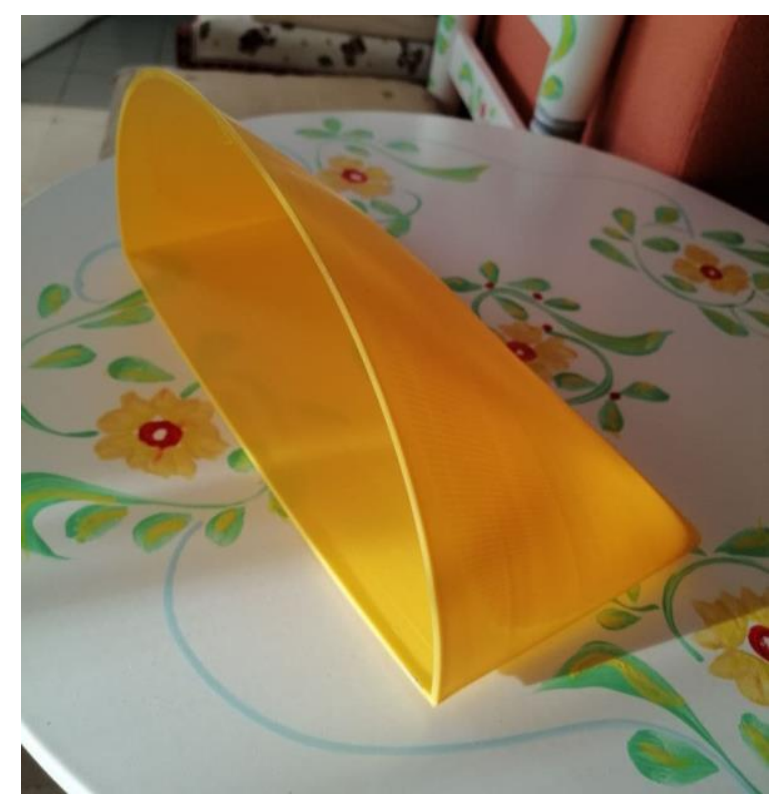

Figure 33. 3D print of a conoid similar to Doganoff's skylight.

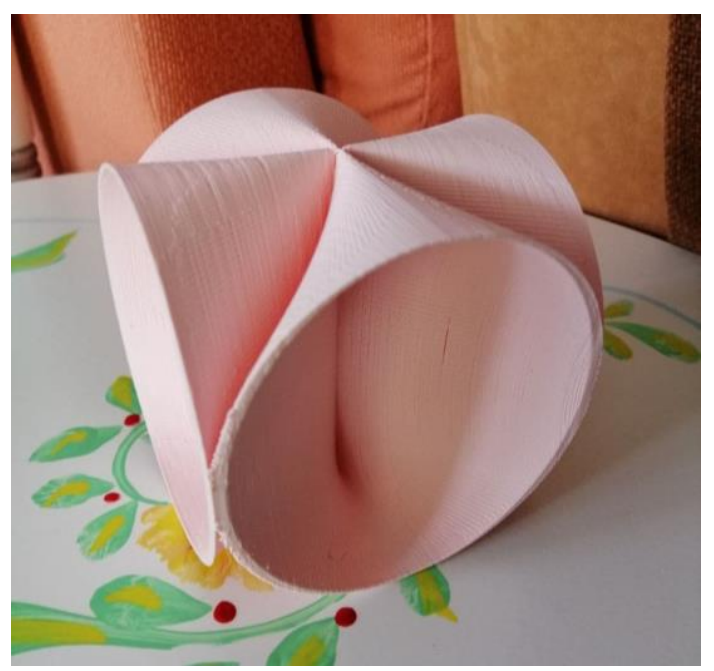

Figure 34. the Dyosphera in 3D print, detail of interlocks.

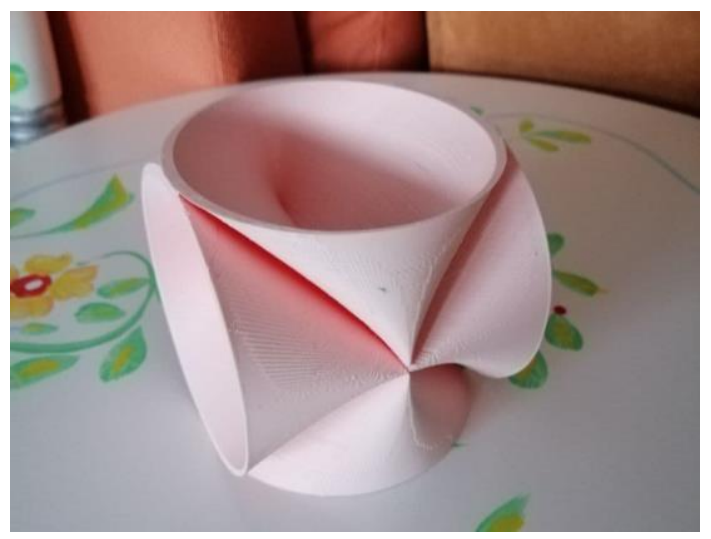

Figure 35. The Dyosphera standing on one of its four bases. 
We believe that the implications of this geometrical advance are far-reaching. Due to its internal logic, it would be suitable for biotechnology [13]. Specially the last development, the Pterasphera being of a tubular nature would be prone to fluid transportation. As a spring-like configuration it conjoins flexibility and balance. In figures 36 and 37 we present examples of possible association and growth in parallel or opposed patterns.

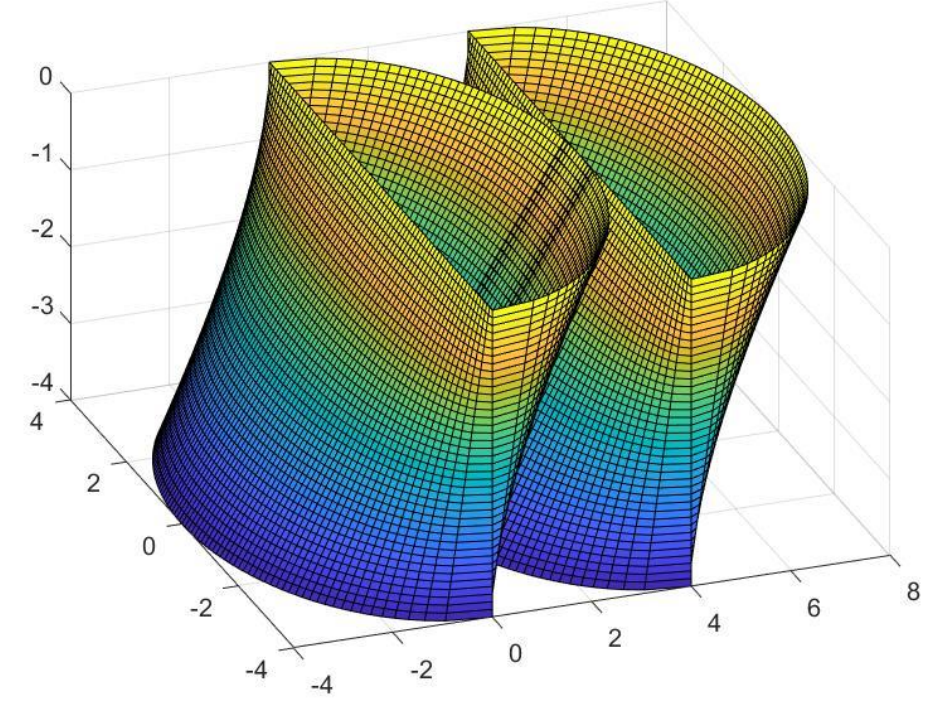

Figure 36. Two parallel Pterasphera tubules

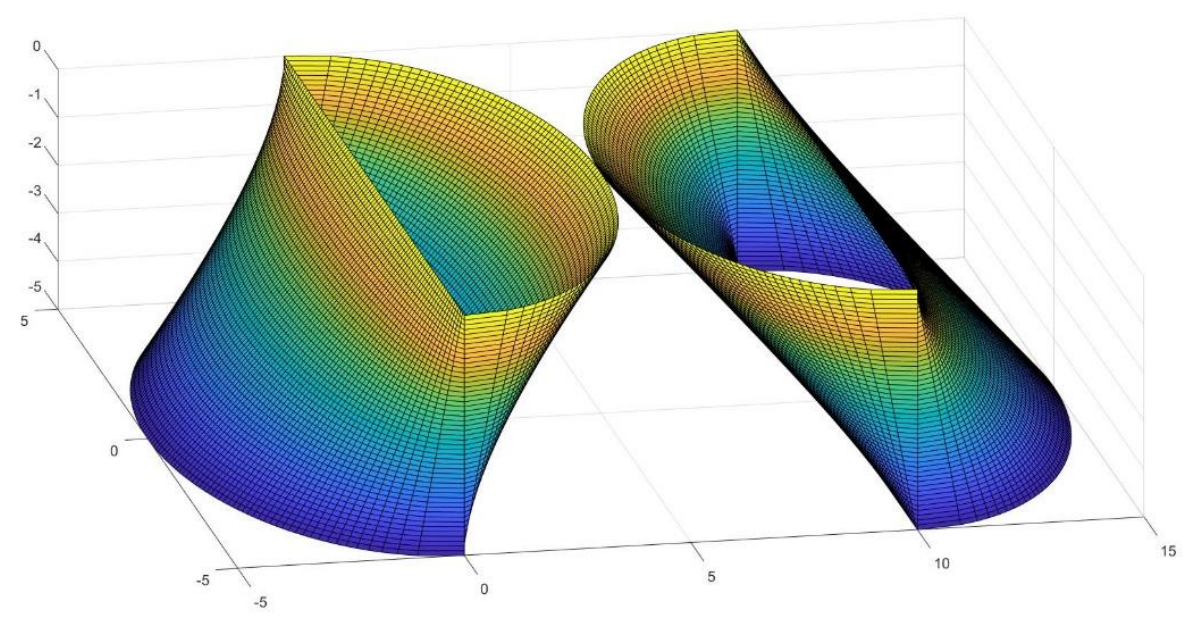

Figure 37. The same tubules opposed

If we analyze the internal section of the tubules (Fig. 38) it is composed of two semi-ellipses of varying sizes, but the span is constant at $\mathrm{R}$, the extreme one is a semi-circumference of radius $\mathrm{R}$ and the middle horizontal section is a complete ellipse of minor axis $\mathrm{R}$ and major axis $2 \mathrm{R}$. 


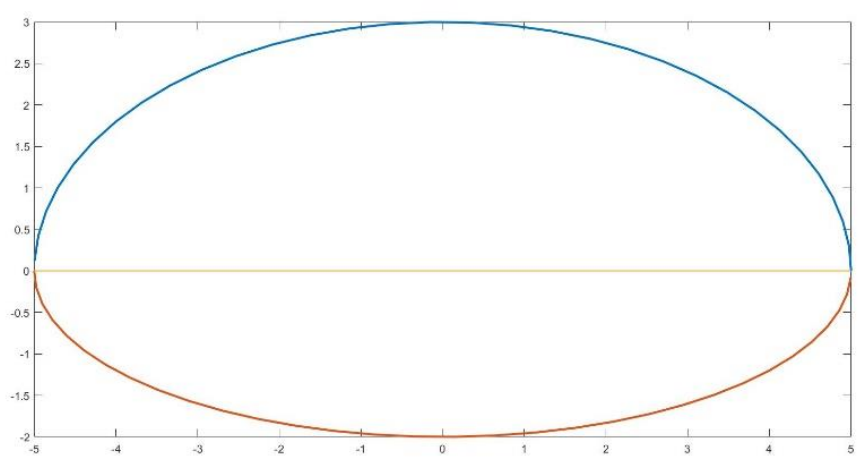

Figure 38. Horizontal section of a Pterasphera of major axis 10 and minor 5

The dimensions of the section are constant but its shape is not and thus the velocity of the fluid inside the tubule can be deftly regulated from the same form. This would offer a clear alternative to reduce the noise level in the ducts or to decant particles in suspension.

The longitudinal central section of the Pterasphera gives a lozenge of horizontal dimensions $\mathrm{R}$ and the sides are inclined to the angle $(\pi / 2-\theta)$, with $\theta=\arctan (\mathrm{L} / \mathrm{R})$. The perpendicular distance between inclined sides is of $d=R \cos \theta$.

This characteristic will facilitate insertion in existing rectangular ducts for example in retrofits. Mass fabrication is also simple since the form allows cuboid molds of middle section over $2 \mathrm{R} \times \mathrm{R} \cos \theta$.

In figure 39 we present an example of vertical growth of the tubules, resembling vegetal pillars.

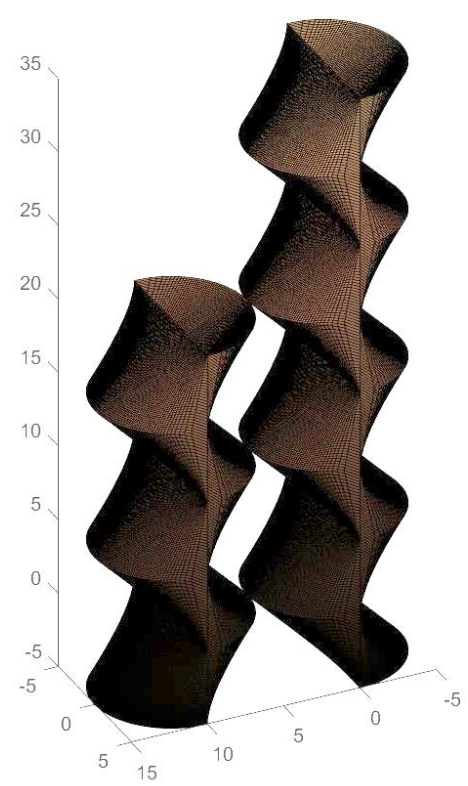

Figure 39. A pair of tubules of different height. 

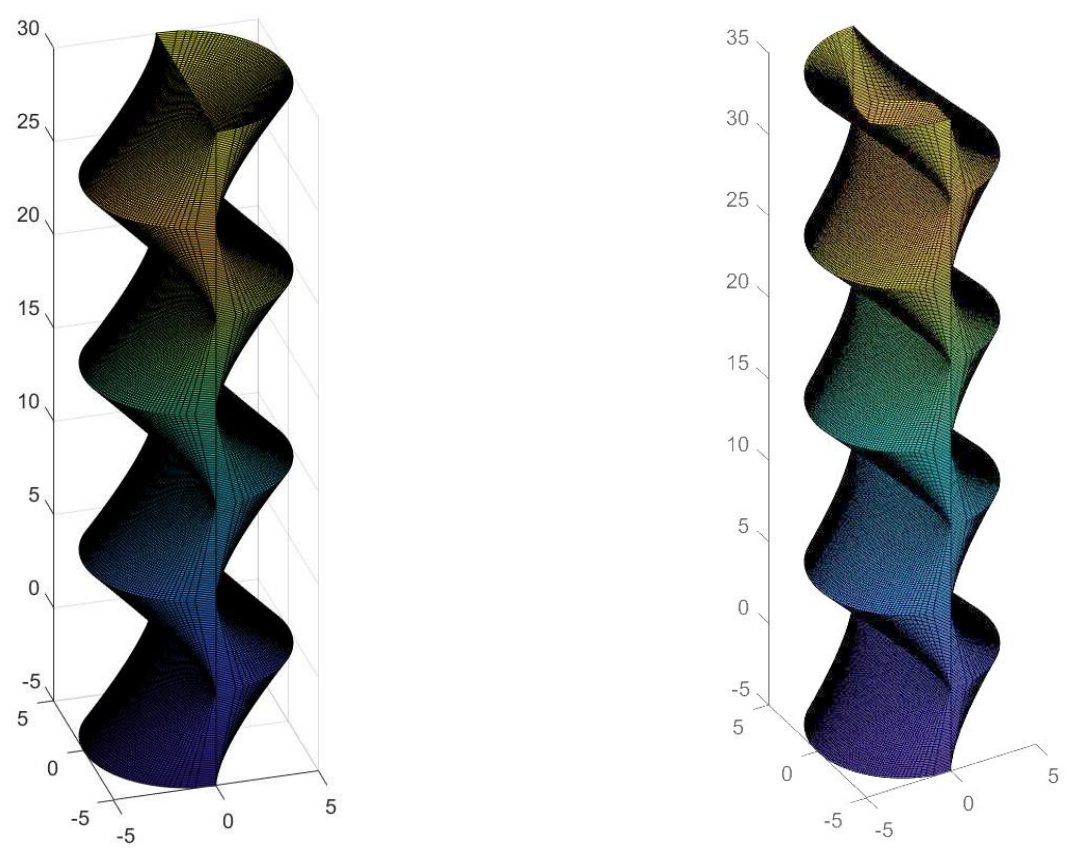

Figure 40. Different perspectives of the tubule.

We decided to explicate this finding, because of the possibilities that it showcases. For instance, in tower buildings. Vertical connections are always feasible at the middle plane of the column, but the external envelop will benefit from the sun tracking or shading properties already exposed combined with new photic materials of variable transparency (Fig. 40). In consequence lighting, thermal and acoustic features will considerably improve the existing conditions.

The structure of the so-conceived tower can be as lightweight as desired due to its inner balance and counterweight. As we have explained previously, alone or better in groups (Fig. 41), it should perform adequately under earthquakes, extreme wind conditions or other unpredictable circumstances.

Diverse technological fields could have a keen interest in the new forms that we have found both for the macro and micro-scales. It is indeed a leap into a sustainable future that we hope to see beyond. 


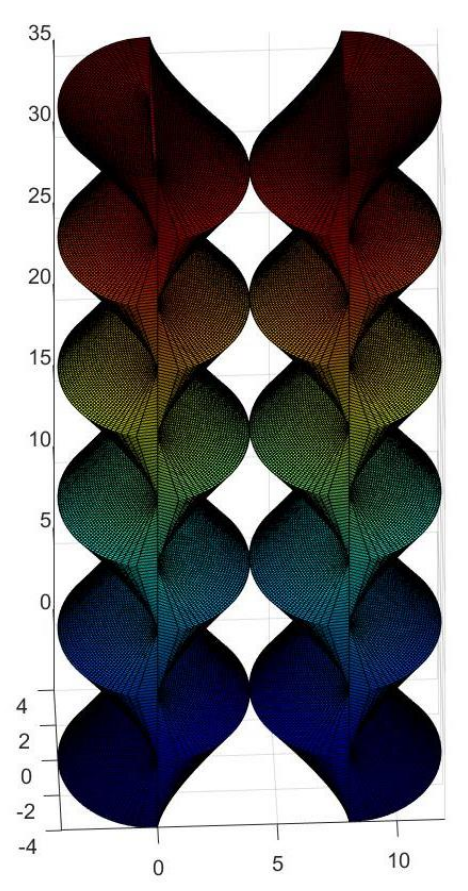

Figure 41

\section{Conclusions and future aims}

We have obtained a new transcendent number that we would call $\psi$, of a very precise nature which provides an accurate approximation of $\pi$. Thanks to Ramanujan's clairvoyant prediction we have found the lateral area of the conoid, a recurrent form in organic structures whose scientific and technical knowledge was insufficient and desired.

We have shown its utility for the design of many types of aeronautical parts and vessels. Due to its particular geometric properties it can both work for heat-storing or dissipation, it performs aptly especially in thermal, luminous and acoustic radiation domains. We have also discussed how the notions that led to this form have evolved since Early Modern Times. All the former make these structures rather sustainable.

Further advantages to outlined are: it enhances the employ of an engineering and architectural form which can save considerable amounts of materials and building time not only when used as cover or roofing in the sense of vaults or domes, but also vertically for cantilevered stadiums and amphitheaters that tend to derive from the cylinder since the Roman Colosseum. 
From the building perspective, easy reinforcement for pre-stressed construction and enhanced structural behavior could be other reasons to use them. Self-shading of the structure and built-in protection from weather phenomena are added values and contribute to its durability.

In the process, we have created no less than four new types of figures which present a high potential in many fields. Especially the last one Pterasphera, owing to its tubular nature can provide interesting developments in biotechnology and fluid-conducting devices.

The repertoire of possibilities derived from this geometric finding and exploration seem to be never ending.

Perhaps the paramount finding hereby exposed is the attribution of a precise meaning for the number $\pi^{2}$, an achievement that leaves us wondering on the notions of $\pi^{3}, \pi^{\mathrm{N}}$ and $\pi$ elevated to infinite power $\infty$.

Author contributions: J.M. Cabeza-Lainez has developed and envisioned the whole work. He programmed all the figures and solved all the equations. All faults in this article are necessarily his.

Funding: This research received no external funding.

Acknowledgments: This article is dedicated to Dr. J. M. Cabeza Arroyo. The author would also like to express remembrance for the late Dr. J. R. Perez de Lama who showed great interest in the early phases of this research. Dr. J. L. Perez de Lama Halcón was helpful in the preliminary 3D fabrication models used by the author to ascertain the hypotheses.

Conflicts of Interest: The author declares none.

\section{References}

1. The Bible. 1 Kings 7:23. "And he made the Sea of cast bronze, ten cubits from one brim to the other; it was completely round. Its height was five cubits, and a line of thirty cubits measured its circumference"

2. Ramanujan, S. Ramanujan's Collected Works, Chelsea, New York, 1962. 
3. Jeffrey, A.; Dai, H. Handbook of Mathematical Formulas and Integrals; Elsevier Academic Press: Burlington, Massachussets, 2008.

4. Weisstein, Eric W. Trascendent Numbers. In Weisstein, Eric W, ed. MathWorld. Wolfram Research.

5. Guarini, C. G. Euclides Adauctus et Methodicus Mathematicaque Universalis. 1671. Torino (Italy).

6. Cabeza-Lainez, J.M. Fundamentos de transferencia radiante luminosa o la verdadera naturaleza del factor de forma y sus modelos de cálculo; Netbiblio; Seville, Spain, 2010.

7. Salguero-Andújar, F.; Cabeza-Lainez, J.-M. New Computational Geometry Methods Applied to Solve Complex Problems of Radiative Transfer. Mathematics 2020, 8, 2176. https://doi.org/10.3390/math8122176

8. Cabeza-Lainez, J.M.; Pulido-Arcas, J.A. New configuration factors for curved surfaces. Journal of Quantitative Spectroscopy and Radiative Transfer 2012, http://dx.doi.org/10.1016/j.jqsrt.2012.10.022

9. Cabeza-Lainez, J. et al. New configuration factor between a circle and a point-plane at random positions. International Journal of Heat and Mass Transfer, 69 147-150, Feb, 2014

10. Salguero Andujar, F.; Rodriguez-Cunill, I.; Cabeza-Lainez, J.M. The Problem of Lighting in Underground Domes, Vaults, and Tunnel-Like Structures of Antiquity; An Application to the Sustainability of Prominent Asian Heritage (India, Korea, China). Sustainability 2019, 11, 5865. https://doi.org/10.3390/su11205865

11. Doganoff, I. Berechnung von Kugelschalen über rechteckigem Grundriß. Mit Berechnungsdiagrammen. Mitteilungen aus dem Institut für Massivbau Hannover. Düsseldorf, Werner Vlg., 1962.

12. Almodovar-Melendo, J.-M.; Cabeza-Lainez, J.-M.; Rodriguez-Cunill, I. Lighting Features in Historical Buildings: Scientific Analysis of the Church of Saint Louis of the Frenchmen in Sevilla. Sustainability 2018, 10, 3352. https://doi.org/10.3390/su10093352.

13. Gómez-Gálvez, Pedro; Vicente-Munuera, Pablo; Tagua, Antonio; Forja, Cristina; Castro, Ana M.; Letrán, Marta; Valencia-Expósito,Andrea; Grima,Clara; Bermúdez-Gallardo, Marina (27 July 2018). "Scutoids are a geometrical solution to three-dimensional packing of epithelia". Nature Communications. 9 (1): 2960. doi:10.1038/s41467-018-05376-1

14. Ramswamy, S.G. Design and Construction of Concrete Shell Roofs, 2004.

15. Doganoff, I.;Hoffmann, C.; Rühle, H. Shell and fold structure roofs out of precast, pre-stressed reinforced concrete elements. 1964.

16. Cabeza-Lainez, J. et al. Rehabilitación del pabellón Plaza de América de la Exposición Universal de 1992. Revista de Edificación. Ecoconstrucción. 1997. Vol. 27. https://hdl.handle.net/10171/16978

17. Cross, H. The Column Analogy. University of Illinois Bulletin. Vol. XXVIII October 14, No.7 1930. 
18. Cabeza-Lainez, J. et al. The Key-role of Eladio Dieste, Spain and the Americas in the Evolution from Brickwork to Architectural Form. Journal of Asian Architecture and Building Engineering. 2009. Vol.8. https://doi.org/10.3130/jaabe.8.355

19. Cabeza-Lainez, J.; Almodovar-Melendo, J.-M.; Dominguez, I. Daylight and Architectural Simulation of the Egebjerg School (Denmark): Sustainable Features of a New Type of Skylight. Sustainability 2019, 11, 5878. https://doi.org/10.3390/su11215878 